\title{
THE TAMAR TROUGH REVISITED: CORRELATIONS BETWEEN SEDIMENTARY BEDS, BASALTS, THEIR AGES AND VALLEY EVOLUTION, NORTH TASMANIA
}

\author{
by F.L. Sutherland, I.T. Graham, S.M. Forsyth, H. Zwingmann and J.L. Everard
}

(with eight text-figures, two plates, nine tables and two appendices)

\begin{abstract}
Sutherland, F.L., Graham, I.T., Forsyth, S.M., Zwingmann, H. \& Everard, J.L. 2006 (30:xi). The Tamar Trough revisited: correlations between sedimentary beds, basalts, their ages and valley evolution, North Tasmania. Papers and Proceedings of the Royal Society of Tasmania 140: 49-72. https://doi.org/10.26749/rstpp.140.49 ISSN 0080-4703. Geoscience, Australian Museum, 6 College Street, Sydney, New South Wales 2010, Australia (FLS*, ITG); Tasmanian Geological Survey, Mineral Resources Tasmania, PO Box 56, Rosny Park, Tasmania 7018, Australia (SMF, JLE); CSIRO Division of Petroleum Resources \& Centre of Excellence in Mass Spectrometry, School of Applied Geology, Curtin University, Western Australia 6102, Australia (HZ). *Author for correspondence.
\end{abstract}

The Tamar Trough, an Early Palaeogene fault structure, contains sedimentary beds and interleaved basaltic flows that infill the structure along its $70 \mathrm{~km}$ length. These infills represent a complex incerplay between sedimentation, channel erosion, eruptive dislocations, and even 'out of trough' diversions of the ancestral Tamar drainage. Several areas of resistant basalt flows remain in the south, upper, middle and lower Tamar reaches. Although some palynological control was known, radiometric dating of previously untested basalts now allows close integration and age-pegging før observed palynological biozones. The $\mathrm{K}$-Ar and Ar-Ar ages of the basalt bodies indicate eruptive events at 47, 33-37 and $25 \mathrm{Ma}$, correlating with Proteacidites asperopolus-Malvacipollis diversus, Nothofagites asperus and Proteacidites tuberculatus biozone age sedimentary beds respectively. Basanite, alkali basalt and hawaiite flows dominate basalt lithology with lesser olivine nephelinite, transitional olivine basalt, olivine tholeiite and quartz tholeiite. Basalt geochemistry suggests derivation from different degrees of partial mantle melting (from 7 to $35 \%$ ), with alkaline and tholeiitic basalts being derived from separate source regions. Most alkaline basalts have high- $\mu$ (HIMU) related trace element signatures, which are absent in the tholeiitic rocks. A basalt plug on the trough margin at Loira gave a Jurassic age and has Jurassic dolerite-like geochemistry. The Tamar sequence suggests that the initial fluvio-lacustrine and later channel-fill sedimentation from 65(?) to 24(?) Ma was then punctuated in places by periods of alkaline volcanism between 47 to $33($ ?) $\mathrm{Ma}$, and alkaline and tholeiitic volcanism between 33 to 24(?) Ma. No Neogene fossils are known, so this later period was probably one of net erosion. These contrasting quiet sedimentary and more volcanic intervals are related here to a tectonic model that involves northerly drift of Victorian and Tasmanian lithosphere over several former Tasman metasomatised mantle plume sources.

Key Words: Tamar Trough, Cenozoic, palynostratigraphy, basalt dating, volcanism.

\section{INTRODUCTION}

Half grabens and grabens form the Tamar Trough, an in-filled structure developed by latest Cretaceous to earliest Cenozoic rifting (Forsyth 1989) that was linked to faulting that formed the Bass Basin (Morrison \& Davidson 1989). This 70-km long, NNW trending fault structure is an offshoot of the Longford sub-basin fault structure (Matthews 1989, Direen \& Leaman 1997). Basalt flows are exposed or encountered in cores drilled within the Trough sequence and these have diverted old river courses, particularly in the south where the South Esk was finally deflected west to enter the Tamar River at Launceston (Carey 1946, Sutherland 1971). The basalt samples were studied in petrographic detail and some contain mantle and/or lower crustal xenoliths and xenocrysts (Sutherland 1969, 1971, Everard 1990, 2001, Sutherland $e t$ al. 2004, and this paper).

Apart from the relationships of the basalt flows to the sedimentary beds, which have some palynological control, the lack of precise radiometric dating on the basalt flows has hampered integration of the sedimentary deposition, volcanism and geomorphic evolution within the trough. An improved understanding of the trough geology has importance, as the interfaces of soft sedimentary beds and resistant basalt flows promote slope movements and hence create problems within housing and construction developments (Turner 1975, Mazengarb 2004).

Extensive palaeobotanical studies exist on Cenozoic sedimentary sequences, including papers of international significance (Hill 1987). Most of the palaeostratigraphic dates, however, are based on biostratigraphy from offshore cores in Bass Strait, from sites over $100 \mathrm{~km}$ away and from very different depositional environments. The present work aims for more direct dating of the local Tamar biostratigraphy.

This paper documents new radiometric dating and petrological data on the basalts along the length of the Tamar Trough. Seven basalts were dated by K-Ar methods, with follow up Ar-Ar dating on three basalts. New analyses of the basalts are presented, as the earlier published petrology lacked trace element data and some further basalt sites were located by later mapping and drilling within the Tamar Trough. This present study is used to correlate the eruptive basaltic episodes with the known palynological zones within the Tamar sediments and thus refine the stratigraphy. The integrated dating will help to establish the drainage evolution that produced the present Tamar River system. Finally, the updated Tamar Trough evolution will be evaluated within the known overall tectonic setting of Tasmania and its Cenozoic volcanism.

\section{GEOLOGICAL SETTING}

The geology of the general area (fig. 1) lies within the Northeast Tasmania 1:250 000 Geological Sheet (McClenaghan et al. 2005), which utilises earlier mapping. The study area from north to south is covered by the 1:63 360 Geological Maps 


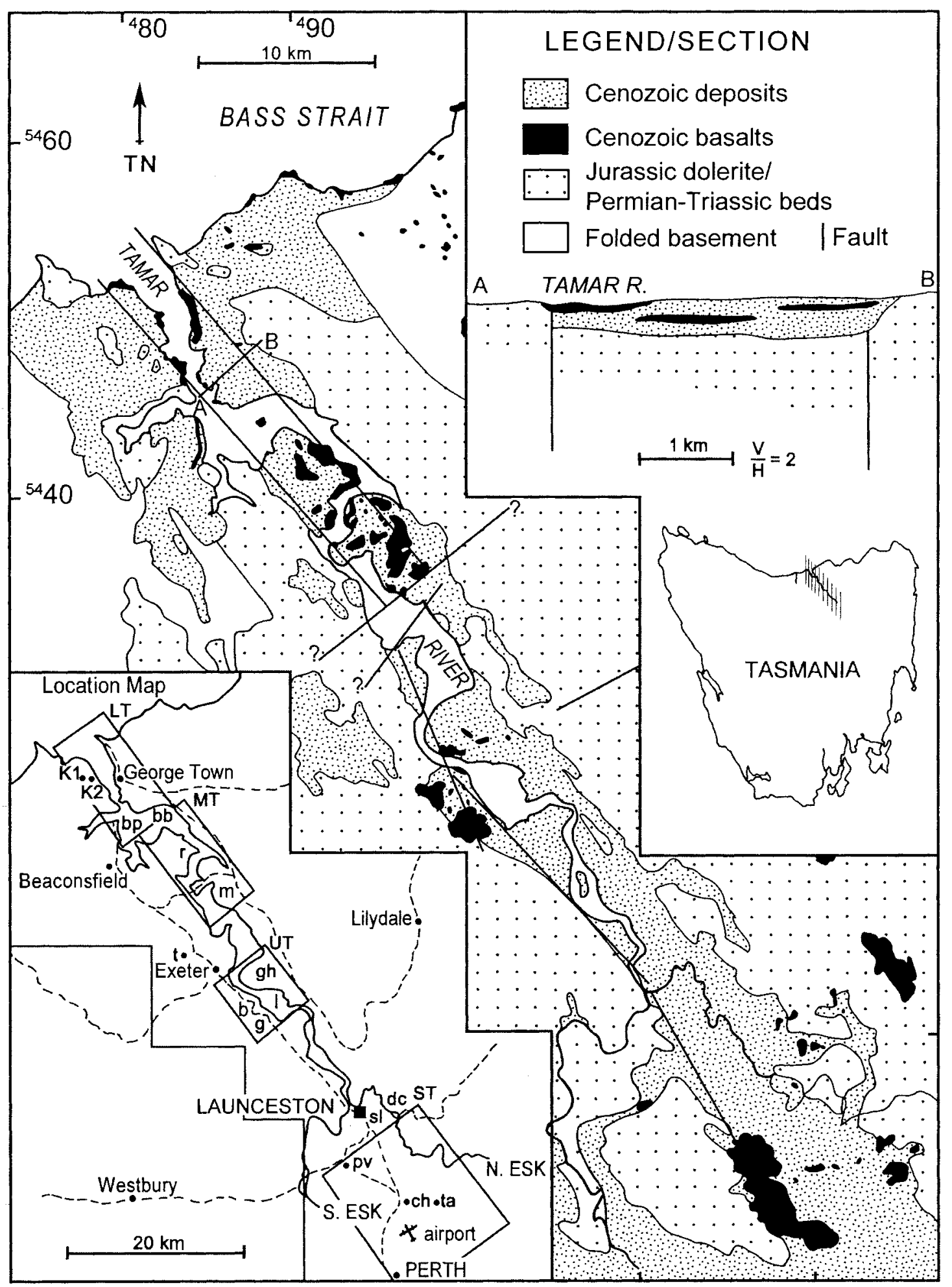

FIG. 1 - General geology, Tamar Trough, showing Cenozoic sedimentary deposits and basalt exposures, within the older rocks. The top right inset shows a stratigraphic legend, a Trough section (based on Leaman et al. 1973) and the Trough location in Tasmania. The bottom left inset shows the main drainages, towns and roads (dashed lines) in the study area, including the South Tamar (ST), Upper Tamar (UT), Middle Tamar (MT) and Lower Tamar (LT) areas. Other cited sites include Cocked Hat Hill (ch), Talisker (ta), Prospect Vale (pv), St Leonards (sl), Distillery Creek (dc), Legana (l), Grindelwald (g), Bradys Lookout (b), Gaunts Hill (gh), Tamerton (t), Murphys Hill (m), Rowella (r), Bell Bay (bb), Beauty Point (bp), Kelso (K).

for Beaconsfield (Gee \& Legge 1971), Frankford (Gulline 1973), Launceston (Longman et al. 1964) and Longford (Blake 1959), the 1:100 000 Longford Basin Map (Matthews 1974 ) and more recent 1:25000 mapping partly in progress. Grid references are based on the Australian Geodetic Datum
\{1966\}, Grid Zone Designation 55G, with 100000 metre square identification letters.

A gravity survey of the Tamar region showed the Tamar structural zone was a prominent down-faulted feature (Leaman et al. 1973). Sheets of Jurassic dolerite intruded into 
Permo-Triassic sedimentary beds dominate the geological framework of the Tamar Trough, with older Palaeozoic basement rocks only exposed beyond the flanks of the structure. Cenozoic sedimentary beds and interbedded basalts occupy the main part of the Trough, along with a range of bauxitic and ferricrete deposits which developed on some horizons. The exposures are partly blanketed by alluvium, talus, landslip and soil deposits related to the more recent erosive process along the Tamar drainage system. Minor wind blown siliceous sands extend in from the Tamar mouth as far as Bell Bay and also occur south of Launceston.

\section{Trough Structure}

The Tamar Trough represents part of the faulting and uplift that led to significant erosion of parts of the Tasmanian highlands during its Late Cretaceous/Early Cenozoic history (Gleadow 1996, O'Sullivan et al. 2000). It links into the structural evolution of the Bass Basin (Cummings et al. 2001), along the Moyston-Tamar Fault Zone, a northwesttrending, broad basement fracture system (Blevin 2003). The tensional rifting that developed along the Tamar Trough lies east of the Mid-Devonian boundary between the East and West Tasmanian terranes (Reed et al. 2002). This boundary was thought to be a transcurrent fault concealed beneath the Tamar Trough (Williams 1979), but more recent ideas suggest that it marks a series of east dipping thrusts coming to the surface between Beaconsfield and Port Sorell (Elliot et al. 1993, Leaman etal. 1994, Reed et al. 2002). The structure lies between a down-warp of the mantle (Moho) under the West Tasmania terrane (Rawlinson et al. 2001) and a change in basement lithosphere under the East Tasmanian terrane (Kennett et al. 2004), but has no direct expression in the underlying lithosphere imaged by Rawlinson \& Kennett (2006). The Moho lies between $29 \mathrm{~km}$ and $32 \mathrm{~km}$ below the Tamar Trough and shallows towards Bass Strait (Clitheroe $e t$ al. 2000). The Tamar region presently shows moderately high surface heat flow (85-95 m W/m-2; O'Sullivan \& Kohn 1997 , O'Neill et al. 2003) and is underpinned by a moderately slow upper mantle shear-wave (thermal) structure (Kennetr 2003, Kennett et al. 2004). The lithosphere here lies on the outskirts of a dormant mantle plume in the asthenosphere under Bass Basin, which acted as a trigger for hotspot volcanism along eastern Australia (Sutherland 2003) and is imaged down to $-650 \mathrm{~km}$ depth (Montelli et al. 2004). The crust here, however, is presently under compression with a mean horizontal stress maximum along a WNW-ESE direction (Clark \& Leonard 2003).

\section{Palynology Zones}

The sedimentary beds within the Tamar Trough date back through several assigned palynological zones, with macroplant fossils only playing a minor role in correlations (Jordan \& Hill 2002). In a general way, the sedimentary units at and below the present Tamar River level become older upstream towards Launceston and younger downstream. Palaeocene beds are found at $60 \mathrm{~m}$ above sea level (ASL) at Launceston, although topographic distribution may be affected by growth faulting. The oldest palynoflora in drill cores can be assigned to the Forcipites longus Zone (Late Cretaceous into Palacocene). South of Launceston, Palaeocene and Early Eocene deposits underlie what may be an erosion surface at the base of the Lower or Middle Nothofagidites asperus Zone with Eocene micro-flora (Stover \& Partridge 1973, Bigwood et al. 1988). Above Rose Rivulet, basalt flows and conglomerates with basalt clasts occur here within or above the current Lower N. asperus Zone (Middle Eocene to Early Oligocene). Palaeocene palynoflora found in the eastern Tamar estuary opposite Legana belong to the Lygistepollenites balmei Zone. In cliffs on the western side at Legana, however, the listed palynoflora can be assigned to the Early Eocene Middle or Upper Malvacipollis diversus Zone (Bigwood et al. 1988). The ages adopted for the palynology zones are those proposed for the offshore Gippsland Basin by A.D. Partridge, which were presented by MacPhail (1999).

The $N$. asperus Zone palynofloras occur north of Legana within the older Early Eocene sedimentary sequence above Windermere and may represent younger channel deposits (Wells 1988), although stratigraphic inversion of palynofloras and potential block slides produce uncertainties in interpretations here. Further north, in the Long ReachEast Arm area, the oldest sedimentary beds found at river level belong to an undifferentiated Upper $M$. diversus Zone to Proteacidites asperopolus Zone interval (Early to Middle Eocene) and include interbedded basalt. Closer to the Tamar mouth, near Bell Bay, the palynofloras under basalt flows lie below sea level (BSL) and belong to Lower-Middle Proteacidites tuberculatus Zone (Oligocene), which occurs within a superimposed channel cut in pre-N. asperus Zone beds. Although the Jurassic dolerite basement is deepest here $(250 \mathrm{~m} \mathrm{BSL})$, the Palaeocene sedimentary interval is either absent or very much reduced in thickness.

The palynology zones used in this study are correlated within a regional geochronology linking the Gippsland Basin sequence to Age (Ma BP), Sea Level Curves, Australian and International Biozonations (spores \& pollens, dinocysts \& acritarchs, benthic foraminifera, planktonic foraminifera, nanoplankton), Magnetic Polarity and the Geological Time Scale (Glenn et al. 1999). Care is needed to translate the Lower Notofagidites asperus zone as used by Stover \& Partridge (1973) into later current usage (Bigwood et al. 1988; MacPhail 1999), where the original L. N. asperus Zone has been divided into new Lower and Middle zones.

\section{BASALT SETTINGS}

The basalt flows and intervening sedimentary beds appear in four principal areas (fig. 1), with each area including centres of volcanism from which flows extended for distances of 5-10 $\mathrm{km}$. Only some feeders are known for the lavas that erupted within the Trough. One basalt plug was mapped outside the Trough at Supply Creek (Gulline 1973) and was sampled in this study as a potential lava source ("Tamerton" plug). Total magnetic intensity images derived from aeromagnetic surveys (with $200 \mathrm{~m}$ line spacings) indicate that although some basalt flows have normal polarity, many are reversely polarised, particularly in the Middle, Upper and South Tamar sections (pl. 1).

Basalt sampled in new exposures and drill cores since the earlier studies, including that intended for age-dating and chemical analysis, was thin-sectioned for detailed microscopic examination (pl. 2) and description (appendix A). Adjacent basalt flows in the Longford Basin (Matthews 1983) were also analysed for comparison with the Tamar Trough basalt flows (appendix B). 

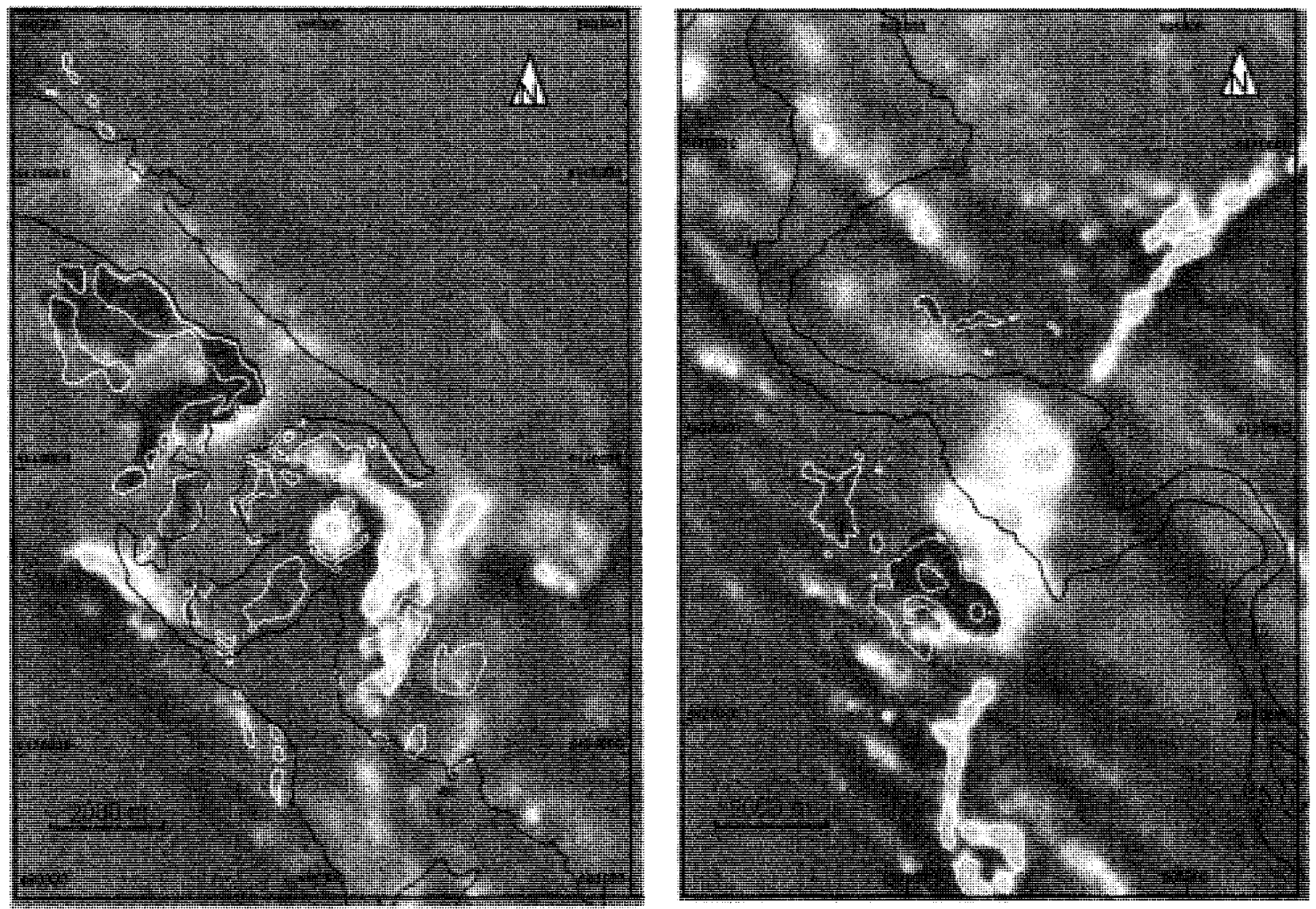

PLATE 1

Tamar Trough total magnetic intensity images, derived from Western Tasmanian Regional Minerals Program aeromagnetic surveys, 200 m line spacing 2001-2002. A. Middle Tamar. B. Upper Tamar. Basalt exposures have white outlines, while the Tamar River has blue outlines. Anomalies that can be linked to basalt include negative anomalies (darker green areas) that suggest reversed polarity of remanent magnetization, while positive anomalies (orange) suggest normal polarity. Some other anomalies related to depth to Jurassic dolerite component of basement are visible in both images. Some strong positive anomalies of uncertain origin may be related to dolerite intrusive structure and composition.

\section{South Tamar Area}

South of Launceston, a massive alkali basalt flow up to 40 $\mathrm{m}$ thick with reverse magnetic polarity extends south from a plug rising to $221 \mathrm{~m}$ ASL at Cocked Hat Hill, Bredalbane. It continues under Launceston airport southward down to 150 $m$ ASL where it diverted the South Esk River course. The plug intrudes strata belonging to the $N$. asperus Zone and the associated flow overlies lignites with a Lower $N$. asperus Zone micro-flora. It is unclear on present exposures whether the flow base rests on an erosional interval.

The Cocked Hat Hill plug (EQ 16105033, $180 \mathrm{~m}$ ASL) was selected as the most significant basalt in the area for $\mathrm{K}-\mathrm{Ar}$ and $\mathrm{Ar}-\mathrm{Ar}$ dating. Several smaller flow and plug remnants of diverse petrology occur northeast of the main flow (Sutherland 1969 and this paper). These include basanites and alkali basalts that erupted from vents around the Talisker-Corra Linn area which infill drainages cut into Early Eocene beds. Basanite resembling that at Talisker forms flow remnants to the northeast and as far south as the South Esk Bridge at Perth (EP 151963, $140 \mathrm{~m} \mathrm{ASL}$ ) and as far west as Newry in drill cuttings (EP 10259735, $140 \mathrm{~m}$ ASL), $11 \mathrm{~km}$ from Talisker. Some olivine nephelinite and glassy basanite remnants lie respectively near Dunedin Farm and Distillery Creek east of St Leonards, whereas olivine tholeiite remnants at $215 \mathrm{~m}$ ASL at Abels Hill near St Leonards overlie quartz gravel beds that rest on Middle-Late Eocene siltstones (Sutherland 1969). Olivine basalt transitional between alkali and tholeiitic types ( $\mathrm{pl}$. 2) overlies clayey sandstone and silty claystone beds at $180 \mathrm{~m}$ ASL in a small valley-fill exposed in the new Bass Highway near Prospect Vale (EQ 104066). Most of these basalts have very fine grained or glassy groundmasses and are not suitable for dating.

\section{Upper Tamar Area}

North of Launceston, massive alkali basalt flows form prominent remnants above Rosevears on the West Tamar and extend from the western shores to $200 \mathrm{~m}$ ASL above Bradys Lookout and onto Grïndelwald plateau above the Loch Lea-Strathlyn area, where water bores indicate a $100 \mathrm{~m}$ thick capping flow. Similar, lesser flow remnants on the opposite eastern shore rise above Windermere to cap Gaunts Hill at $160 \mathrm{~m}$ ASL. The number of flows is uncertain as the basalts all show reverse magnetic polarity (pl. 1), but two distinct basalt types were recognised in western exposures, coarse alkali basalt-hawaiite (pl. 2B) and fine grained basanite (pl. 3C). Excavations in 2003 at Rosevears Estate winery at 120 

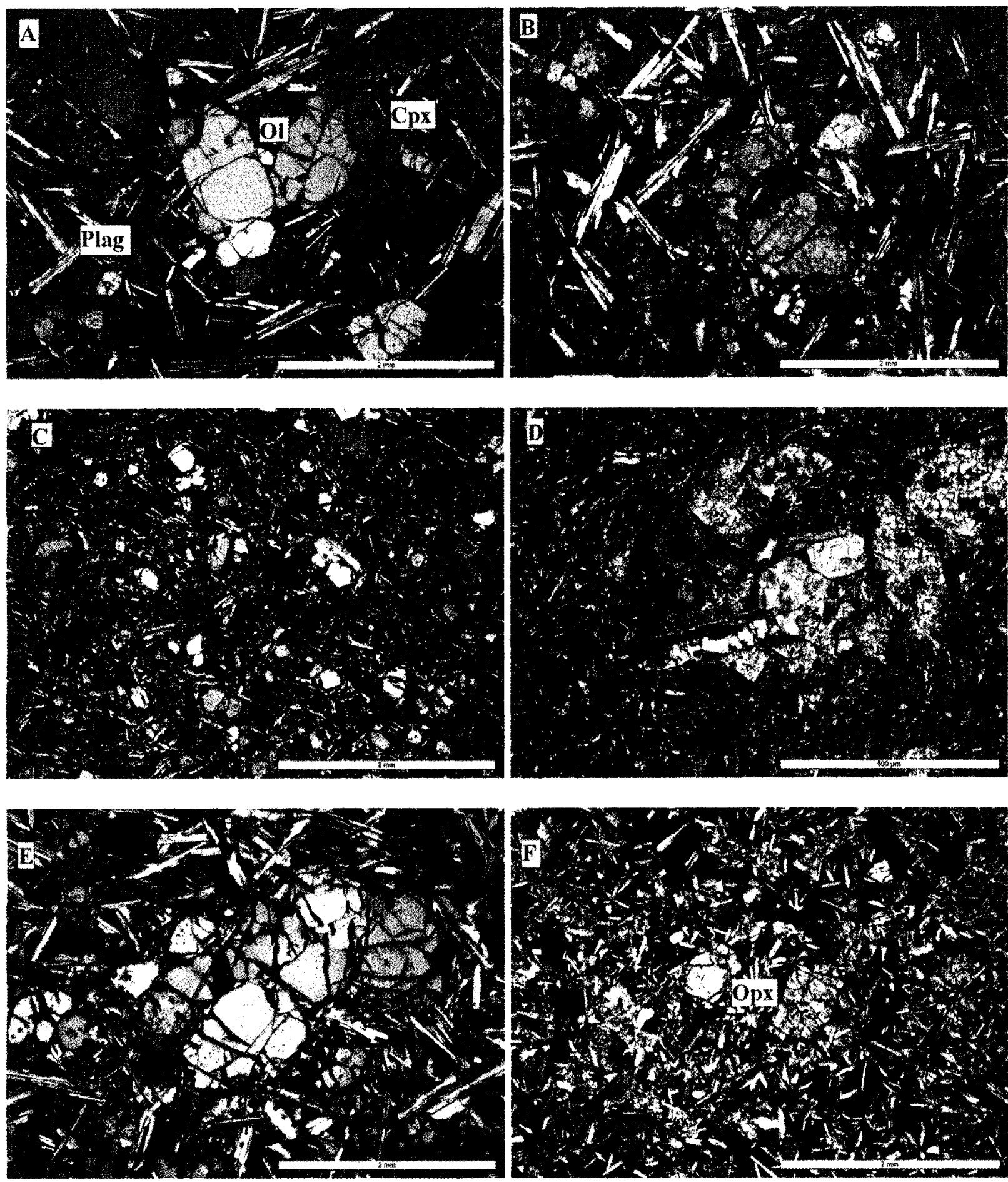

PLATE 2

Photomicrographs of Tamar basalt thin sections. A. Prospect Vale, transitional olivine-basalt. B. Coarse alkali olivine basalt, Rosvears Estate, Strathlyn. C. Basanite, Rosevears Estate, Strathlyn. D. Chilled vesicular hawaiite, Rowella DDH 1. E. Coarse hawaiite, Rowella DDH 2. F. Quartz tholeiite, Tamerton' basaltic' plug. All microphotographs taken with crossed polars. All scales are $2 \mathrm{~mm}$, except for $D$ at $0.5 \mathrm{~mm}$. Photos: I. T. Graham.

$\mathrm{m}$ ASL exposed a vertical contact of fine grained basanite with vertical cooling columns abutting slightly weathered coarse alkali basalt (EQ 5007005423200 ). Underlying strata here lack palynological assignment, but Early Eocene beds are known to the south and east at Legana and Gaunts Hill. The Gaunts Hill basalt may occupy a channel cut into the older sedimentary beds as an explanation for the $N$. asperus Zone deposits found above Windermere. To resolve the age of the Rosevears basalts and the underlying strata, and to assist correlations with the Windermere-Gaunts Hill section, coarse and fine grained basalts in the Loch Lea-Grïndelwald section were selected for $\mathrm{K}-\mathrm{Ar}$ and $\mathrm{Ar}-\mathrm{Ar}$ dating. 


\section{Middle Tamar Area}

Basalt crops out between Deviot and Rowella on the western Tamar shore and from Craigburn to East Arm on the eastern shore. The exposures extend from river level up to $180 \mathrm{~m} \mathrm{ASL}$ on Murphys Hill, east of Craigburn and include hawaiite, basanite, olivine nephelinite and a transitional olivine basalt plug (Sutherland 1969). A western massive hawaiite flow descends below river level and was drilled at Rowella (DDH 1 and 2), where it lies within strata of Upper $M$. diversus Zone or $P$. asperopolus Zone age. The basalt top showed some vesicularity, with no obvious baking of the overlying mudstone (DDH1), suggesting it forms a chilled flow top (pl. 2D) of a coarse flow (pl. 2E), rather than representing an intrusion. This makes it the oldest basalt recorded in the Tamar Trough, marking the drill core (DQ 930413) as a prime target for K-Ar and Ar-Ar-dating.

The eastern flows occupy channels cut into similar aged beds to those dated at Rowella. The massive hawaiite that caps Murphys Hill through to East Arm, however, is normally magnetised, unlike the underlying Spring Bay olivine nephelinite and the Rowella hawaiite, which show reverse polarity (pl. 1). The Murphys Hill hawaiite also abuts a dolerite-clast conglomerate that contains rare basalt clasts, so the evidence clearly suggests different episodes of hawaiite extrusion. Dolerite-clast conglomerates form extensive lenses within the sedimentary beds east of the basalts from East Arm to Hillwood, which may indicate a re-routing of palaeodrainage. The upper hawaiite exposed in the Batman Highway road cuts was sampled for K-Ar dating (DQ 965392, $100 \mathrm{~m} \mathrm{ASL}$ ) not only to check the separate hawaiite ages, but also to provide some age control on dolerite-clast conglomerate deposition.

A small 'basaltic' plug (pl. 2F) mapped near "Tamerton" above Supply River (DQ 920302, 80 m ASL) was sampled for K-Ar dating in case it formed a potential eruptive site for flows to enter the Tamar Valley through an erosional low south of Loira.

\section{Lower Tamar Area}

Low-lying basalt flow remnants extend along the Tamar shores from south of Bell Bay on the east and from south of Beauty Point on the west ro outlet at Tamar Heads between Low Head and West Head. The flows include a lower hawaiite with a base that descends from $>46 \mathrm{~m}$ ASL to $>18 \mathrm{~m} \mathrm{BSL}$. The base was intersected in Bell Bay drill cores where it overlies unweathered sedimentary beds related to the Lower-Middle P. tuberculatus Zone (Oligocene). The flow was also drilled south of George Town (Tamar Avenue DDH) where $20 \mathrm{~m}$ of basalt was intersected overlying sedimentary beds at $-5 \mathrm{~m}$ ASL (DQ 4850005447000 ). A sample of this core retained in the Tasmanian Museum collection was selected for K-Ar dating to test the palynological correlation.

An upper transitional olivine basalt descends from a base at $>30 \mathrm{~m}$ at Bell Bay to $-15 \mathrm{~m} \mathrm{ASL}$ at Beauty Point. It overlies interbasaltic strata and possibly continues through Kelso, west of the lower basalt, as similar olivine basalt was intersected in Kelso Drill Hole DDH 2 (DQ 481400 5449100 ) between 4.4 to $7.3 \mathrm{~m}$ depth where it overlies $7 \mathrm{~m}$ of carbonaceous clay and silrstones and deeper flows (Everard 1990). The Kelso drill holes penetrated the deeper flows between 21 to $60 \mathrm{~m}$ depth (DDH 1) and 14 to $33 \mathrm{~m}$ depth (DDH 2) where they show a distinct glassy nature
(Everard 1990). These basaltic flows are interbedded with thin tuffs, tuffaceous sediments and non-volcanic beds and indicate multiple explosive eruptions. The basal beds $(23 \mathrm{~m}$ thick in DDH 1,9 $\mathrm{m}$ thick in DDH 2) contain decomposed dolerite fragments before solid dolerite is reached at $85 \mathrm{~m}$ (DDH 1) and $41 \mathrm{~m}$ (DDH 2) depth. Although the basalt samples were unsuitable for radionetric dating, the rare carbonaceous sediments provide some palynological control for these sections.

Samples from DDH 1 (DQ 819489) from horizons (54.8 $\mathrm{m}$ depth) between the deeper flows and from $\mathrm{DDH}$ 2 (12.5 $\mathrm{m}$ depth) from horizons between the highest and deeper flows contain spore assemblages no older than the Lower $P$. tuberculatus Zone. The lack of species that first appear in the base of the Middle P. tuberculatus Zone in the Gippsland Basin sequence suggests an Oligocene age for the Kelso sections, in keeping with the Lower-Middle $P$. tuberculatus Zone age for the Bell Bay basalt section.

\section{ANALYTICAL METHODS}

\section{K-Ar Analysis}

The $\mathrm{K}$-Ar isotopic age determinations were made at CSIRO Petroleum, Bentley, Western Australia (table 1). Samples were split and crushed in a ring grinder with chrome steel barrel into $a<500 \mu \mathrm{m}$ fraction. The $\mathrm{K}$ content was determined by atomic absorption spectrophotometry (Varian AA20) using Cs at a concentration of $1000 \mathrm{ppm}$ for ionisation suppression. Sample aliquots were dissolved with hydrofluoric and nitric acid (Heinrichs \& Herrmann 1990) and solutions were diluted for the atomic absorption analysis. Ar isotopic determination followed Bonhomme et al. (1975) with the samples pre-heated to minimise atmospheric Ar adsorbed onto the mineral surfaces. Argon was extracted from the mineral fraction by fusing the sample with a vacuum line serviced by an on-line ${ }^{38} \mathrm{Ar}$ spike pipette. The isotopic composition of the spiked Ar was then measured using an on-line VG3600 mass spectrometer. The ${ }^{38} \mathrm{Ar}$ spike was calibrated against the standard biotite GA1550 (McDougall \& Roksandic 1974). After fusion of the sample in a resistance furnace, the released gases were purified using a $\mathrm{Cu}_{2} \mathrm{O}$ getter and then two Ti getters. Blanks for the extraction line and mass spectrometer were systematically determined and the mass discrimination factor was determined periodically by airshots. During analysis, international standards provided calibration. The error for the Ar analysis is below $1 \%$. The $\mathrm{K}-\mathrm{Ar}$ calculation used constants after Steiger \& Jäger (1977). The age uncertainty includes the errors during sample weighting, ${ }^{38} \mathrm{Ar} /{ }^{36} \mathrm{Ar}$ and ${ }^{40} \mathrm{Ar} /{ }^{38} \mathrm{Ar}$ measurements and $\mathrm{K}$ analysis.

\section{${ }^{40} \mathrm{Ar}-{ }^{39} \mathrm{Ar}$ analysis}

This follows typical ${ }^{40} \mathrm{Ar}-{ }^{-39} \mathrm{Ar}$ dating procedures (McDougall \& Harrison 1999). A concentrate of separated feldspar was cleaned and wrapped in aluminium foil along with biotite standard HD-B1 (24.21 $\pm 0.32 \mathrm{Ma}$; Hess \& Lippolt 1994) to monitor the neutron flux gradient. The package was irradiated in the McMaster University Nuclear Reactor, Hamilton, Canada.

For infra-red laser step-heating, the mineral separate was baked to remove adsorbed atmospheric argon. A $110 \mathrm{~W}$ Spectrum Laser system continuous wave Nd-Y-Al-garnet 
$(\sigma=1064 \mathrm{~nm})$ laser was used to treat the sample. After removing active gases the remaining noble gases were equilibrated into a Mass Analyser Product 215-50 static mass spectrometer (MS) with a multiplier detector. The $\mathrm{Ar}$ sensitivity of the MS multiplier detector was $2.71 \times 10^{-10}$ $\mathrm{cm}^{3} /$ Volt. A Lab View program was used to measure isotope peak masses from 35-41 (10 times) in sequence lasting 15 minutes, via a peak hopping routine. Blank analyses were run before and after.

Age calculation (table 2) involved extrapolating peak intensities back to the inlet time and correcting for extraction line blanks, MS backgrounds, MS discrimination $\left({ }^{40} \mathrm{Ar} /{ }^{36} \mathrm{Ar}\right.$ ratio of 281.0) and reactor interference. The $\mathrm{Ca}$ and $\mathrm{K}$ correlation factors in the data reduction were ${ }^{39} \mathrm{Ar} /{ }^{37} \mathrm{Ar}_{\mathrm{Ca}_{1}}=$ $0.00065,{ }^{36} \mathrm{Ar} /{ }^{37} \mathrm{Ar}_{\mathrm{Ca}}=0.000255$ and ${ }^{40} \mathrm{Ar} /{ }^{36} \mathrm{Ar}_{\mathrm{K}}=0.0015$. Errors quoted are $1 \sigma$ and $A r-A r$ ages (fig. 2) were calculated using decay constants of Steiger \& Jäger (1977). Weighted mean ages were calculated ( $2 \sigma$ errors) using the Ludwig (2001) Isoplot program.

\section{Whole rock chemistry}

The basalt nomenclature used here follows that for Australian basalts defined by Johnson (1989); while the alternative recommended International Union of Geological Science nomenclature (Le Maitre 1989) is covered by using the petrological fields in figure 3. The basalts are dominantly slightly evolved alkali basalts and hawaiites, with subordinate olivine nephelinites, basanites, transitional olivine basalts, olivine tholeiites and quartz tholeiites.

Major and minor elements of basalt samples, listed in table 3 and for Kelso tholeiite in table 5, were made on fresh samples crushed in a steel jaw crusher and determined by $\mathrm{X}$-ray fluorescence (XRF) methods on glass discs prepared by standard techniques at Mineral Resources Tasmania (MRT) Laboratories, Hobart. FeO was determined by titration and CIPW norms were calculated for the basalts using a $\mathrm{Fe}_{2} \mathrm{O}_{3}$ ' $\mathrm{FeO}$ ratio of 0.2 as recommended by Johnson (1989). The $\mathrm{CO}_{2}$ was determined by gravimetric methods, while $\mathrm{H}_{2} \mathrm{O}(+)$ was calculated from loss-on-ignition (LOI) after adjusting for $\mathrm{FeO}, \mathrm{CO}_{2}$ and $\mathrm{SO}_{3}$. The trace element values come from XRF analyses using conventional methods and rock standards on pressed pellets. The other major, minor and trace elements for basalts in table 4 and the "Tamerton" tholeiite in table 5 were analysed at the XRF and XRD Facility, University of Pretoria, South Africa. Samples were ground in a tungsten carbide mill, roasted at $1000^{\circ} \mathrm{C}$ to determine LOI, then fused with lithium borate into glass beads which were analysed by XRF using an ARL $9400 \mathrm{X}$ $1(+)$ spectrometer set up. Another aliquot of sample was pressed into a powder briquette for selected trace element analyses. Precisions for XRF major element oxides (table 4) were $\mathrm{SiO}_{2}$ less than $0.3 \%, \mathrm{TiO}_{2}<5 \%, \mathrm{Al}_{2} \mathrm{O}_{3}<1 \%, \mathrm{Fe}_{2} \mathrm{O}_{3}$ $<3 \%, \mathrm{MnO}<17 \%, \mathrm{MgO}<7 \%, \mathrm{CaO}<12 \%, \mathrm{Na}_{2} \mathrm{O}<2 \%$, $\mathrm{K}_{2} \mathrm{O}<1 \%, \mathrm{P}_{2} \mathrm{O}_{5}<4 \%$ and LOI $3 \%$ and for XRF trace elements were $\mathrm{Ni}<1 \%, \mathrm{Cr}<4 \%$, Co $<16 \%$, Sc $<1 \%$, V $<7 \%, \mathrm{Y}<11 \%, \mathrm{Rb}<1 \%$, Sr $<2 \%, \mathrm{Ba}<1 \%, \mathrm{Zr}<8 \%, \mathrm{Nb}$ $<15 \%$, La $<15 \%$, Ce $<1 \%$, Pb $<2 \%$, Ga $<10 \%$, Th $<12 \%$, $\mathrm{U}<1 \%, \mathrm{Cu}<25 \%, \mathrm{Zn}<1 \% . \mathrm{Mg}$ values were calculated as $100 \mathrm{Mg} / \mathrm{Mg}+\mathrm{Fe}^{2+}\left(\right.$ at $\left.\mathrm{Fe}_{2} \mathrm{O}_{3} / \mathrm{FeO}=0.2\right)$.

The trace and rare earth element (REE) values (table 4) were measured by Inductively Coupled Plasma-Mass
Spectrometry (ICP-MS) techniques at the Geology Department, University of Cape Town, Rondebosch, South Africa. Dissolution of samples used a standard acid digestion procedure before analysis on a Perkin Elmer Sciex Elan 6000 ICP-MS. The instrument operating conditions were optimised to minimise the formation of doubly charged ions and oxides. Interference corrections were made for isobaric interferences and doubly charged ion and oxide interferences (particularly on REE). Both within-run and duplicate analysis precisions were mostly between $2 \%$ relative and always $<$ than 3\% relative (1 sigma).

\section{RESULTS}

\section{Geochronology}

The K-Ar dates (table 1) provide a wide range of ages. Some are minimum results due to some late-stage glass or alteration in those rocks. However, the dates clearly establish several well-separated basaltic events within the Tamar Trough. The youngest dated basalt is represented by the Late Oligocene age for the Lower Tamar hawaiite at George Town (24.7 Ma). It underpins the Bell Bay-Beauty Point basalt sequence and is

\section{TABLE 1}

\section{K-Ar dating results, Tamar Trough basalts}

\begin{tabular}{lccccc}
\hline $\begin{array}{l}\text { Sample no./ } \\
\text { (Rock type) }\end{array}$ & $\begin{array}{c}\mathrm{K} \\
(\%)\end{array}$ & $\begin{array}{c}\text { Rad }{ }^{\mathrm{N0}} \mathrm{Ar} \\
(\mathrm{mole} / \mathrm{g})\end{array}$ & $\begin{array}{c}\text { Rad }{ }^{40} \mathrm{Ar} \\
(\%)\end{array}$ & $\begin{array}{c}\text { Age } \\
(\mathrm{Ma})\end{array}$ & $\begin{array}{c}\text { Error } \\
(\mathrm{Ma})\end{array}$ \\
\hline $\begin{array}{l}\text { Y1295 (TM) } \\
\text { Hawaiite }\end{array}$ & 0.88 & $9.8948 \times 10^{-11}$ & 43.16 & 24.72 & \pm 0.53 \\
$\begin{array}{l}\text { DR16808 } \\
\text { Hawaiice }\end{array}$ & 1.04 & $5.3408 \times 10^{-11}$ & 45.04 & $29.37^{*}$ & \pm 0.87 \\
$\begin{array}{l}\text { DR10499 } \\
\text { Hawaiite }\end{array}$ & 1.05 & $5.9784 \times 10^{-11}$ & 64.71 & $32.53^{*}$ & \pm 0.67 \\
$\begin{array}{l}\text { DR 17070 } \\
\text { Basanite }\end{array}$ & 1.15 & $5.9291 \times 10^{-11}$ & 71.73 & $33.74^{*}$ & \pm 0.70 \\
ROW (TMR) & 0.83 & $6.8126 \times 10^{-11}$ & 61.10 & 46.66 & \pm 1.02 \\
Hawaiite & & & & & \\
$\begin{array}{l}\text { TAM (AM) } \\
\text { Tholeiice }\end{array}$ & 0.71 & $2.0478 \times 10^{-10}$ & 91.07 & $159.28^{*}$ & \pm 3.17 \\
\hline
\end{tabular}

* Probable minimum age due to presence of minor mesostasis, glass or alteration.

TM (Tasmanian Museum sample), TMR (Tasmanian Mineral Resources sample), DR and (AM) Samples are Australian Museum Collection material.

Y1295 (TM). Flow, Tamar Avenue DDH, Georgetown (Beaconsfield DQ 85034469).

DR16808. Plug, Cocked Hat Hill, $180 \mathrm{~m}$ ASL (Longford EQ 16150330).

DR10499. Flow, Batman Bridge Highway, 100 m ASL (Beaconsfield DQ 96503920)

DR17070. Flow, Loch Lea, West Tamar Road, 75 m ASL (Launceston EQ01052325).

ROW (TMR). Flow, Rowella DDH2, $104.5 \mathrm{~m}$ depth (Beaconsfield DQ 93004130).

TAM (AM). Plug, "Tamerton", Supply River, 80 m ASL (Frankford DQ 920302). 
TABLE 2

${ }^{40} \mathrm{Ar}-{ }^{39} \mathrm{Ar}$ analyses, Tamar Trough basalts

\begin{tabular}{|c|c|c|c|c|c|c|c|c|}
\hline Step & Age (Ma) & ${ }^{40} \mathrm{Ar}{ }^{*} / 39 \mathrm{Ar}$ & ${ }^{40} \mathrm{Ar} /{ }^{39} \mathrm{Ar}$ & ${ }^{38} \mathrm{Ar}{ }^{*} / 39 \mathrm{Ar}$ & ${ }^{37} \mathrm{Ar}^{*} / 39 \mathrm{Ar}$ & ${ }^{39} \operatorname{Ar}\left(10^{-12} \mathrm{~cm}^{3}\right)$ & ${ }^{40} \mathrm{Ar}^{*} \%$ & ${ }^{36} \mathrm{Ar} /{ }^{39} \mathrm{Ar}$ \\
\hline \multicolumn{9}{|c|}{ Pegmatitic hawaiite, Grïndelwald plateau, Y1205 (Feldspar separate $459 \mathrm{KF}$ ). } \\
\hline 1. & $30.07 \pm 1.50$ & $4.75 \pm 0.24$ & $6.33 \pm 0.06$ & $0.01637 \pm 0.00039$ & $3.50273 \pm 0.39007$ & $3.48 \pm 0.007$ & 24.90 & $0.39007 \pm 0.00533$ \\
\hline 2. & $37.13 \pm 0.31$ & $5.88 \pm 0.04$ & $7.19 \pm 0.01$ & $0.01637 \pm 0.00007$ & $3.72159 \pm 0.06819$ & $20.60 \pm 0.014$ & 18.23 & $0.06819 \pm 0.00444$ \\
\hline 3. & $36.59 \pm 0.49$ & $4.80 \pm 0.07$ & $7.08 \pm 0.01$ & $0.01323 \pm 0.00013$ & $4.25931 \pm 0.02558$ & $20.20 \pm 0.013$ & 18.17 & $0.02558 \pm 0.00435$ \\
\hline 4. & $39.01 \pm 1.99$ & $6.18 \pm 0.32$ & $7.48 \pm 0.05$ & $0.01523 \pm 0.00071$ & $3.59482 \pm 0.10594$ & $3.83 \pm 0.006$ & 17.33 & $0.10594 \pm 0.00439$ \\
\hline 5. & $32.16 \pm 2.33$ & $5.09 \pm 0.37$ & $6.27 \pm 0.01$ & $0.01416 \pm 0.00025$ & $2.96145 \pm 0.03374$ & & & $0.03374 \pm 0.00401$ \\
\hline 6. & $41.27 \pm 9.38$ & $6.55 \pm 1.50$ & $7.78 \pm 0.02$ & $0.01149 \pm 0.00102$ & $3.59157 \pm 0.13657$ & $1.33 \pm 0.003$ & 15.85 & $0.13657 \pm 0.00417$ \\
\hline 7. & $40.08 \pm 1.38$ & $6.35 \pm 0.22$ & $7.60 \pm 0.03$ & $0.01430 \pm 0.00073$ & $3.58780 \pm 0.13927$ & $1.85 \pm 0.003$ & 16.41 & $0.13927 \pm 0.00422$ \\
\hline 8. & $36.66 \pm 1.11$ & $5.81 \pm 0.17$ & $8.12 \pm 0.03$ & $0.01382 \pm 0.00059$ & $3.74168 \pm 0.07911$ & $2.31 \pm 0.006$ & 28.50 & $0.07911 \pm 0.00784$ \\
\hline 9. & $37.57 \pm 0.20$ & $5.95 \pm 0.01$ & $7.12 \pm 0.01$ & $0.01546 \pm 0.00028$ & $3.07406 \pm 0.01922$ & $9.71 \pm 0.014$ & 16.43 & $0.01922 \pm 0.00396$ \\
\hline \multicolumn{6}{|c|}{ Total gas age $36.73 \pm 3.38 \mathrm{Ma}$} & 68.70 & & \\
\hline
\end{tabular}

Plateau Ar-Ar age $36.98 \pm 0.22 \mathrm{Ma}$ with $74.8 \%$ of ${ }^{39} \mathrm{Ar}$

\section{Hawaiite, Cocked Hat Hill, CCH (TMR) (Feldspar separate 414 KF)}

\begin{tabular}{lllllllll} 
1. & $30.68 \pm 0.68$ & $4.85 \pm 0.10$ & $49.59 \pm 0.30$ & $0.05622 \pm 0.00032$ & $5.81723 \pm 0.24164$ & $10.939 \pm 0.005$ & 90.22 & $0.15141 \pm 0.00089$ \\
2 & $24.92 \pm 0.17$ & $3.93 \pm 0.02$ & $50.45 \pm 0.07$ & $0.04391 \pm 0.00006$ & $6.86041 \pm 0.11141$ & $2.026 \pm 0.003$ & 92.20 & $0.15742 \pm 0.00021$ \\
3. & $32.07 \pm 2.32$ & $5.07 \pm 0.37$ & $58.92 \pm 0.09$ & $0.04858 \pm 0.00024$ & $9.91998 \pm 0.95496$ & $11.961 \pm 0.018$ & 91.39 & $0.18224 \pm 0.00127$ \\
4. & $31.59 \pm 3.95$ & $5.00 \pm 0.63$ & $44.01 \pm 0.06$ & $0.03845 \pm 0.00029$ & $9.52268 \pm 1.01863$ & $22.918 \pm 0.029$ & 88.65 & $0.13202 \pm 0.00214$ \\
5. & $31.82 \pm 4.26$ & $5.03 \pm 0.68$ & $46.97 \pm 0.06$ & $0.04026 \pm 0.0030$ & $10.48736 \pm 1.07583$ & $21.405 \pm 0.024$ & 89.28 & $0.14191 \pm 0.00230$ \\
6. & $32.77 \pm 5.91$ & $5.18 \pm 0.94$ & $35.63 \pm 0.08$ & $0.03284 \pm 0.00055$ & $9.44133 \pm 1.55320$ & $15.295 \pm 0.034$ & 85.45 & $0.10302 \pm 0.00320$ \\
7. & $37.28 \pm 3.37$ & $5.90 \pm 0.54$ & $27.74 \pm 0.02$ & $0.02667 \pm 0.00031$ & $6.44878 \pm 0.89273$ & $26.652 \pm 0.017$ & 78.72 & $0.07390 \pm 0.00182$ \\
8. & $35.48 \pm 2.35$ & $5.62 \pm 0.37$ & $22.29 \pm 0.02$ & $0.02396 \pm 0.00025$ & $5.13939 \pm 0.68285$ & $25.414 \pm 0.014$ & 74.80 & $0.05641 \pm 0.00127$ \\
9. & $38.57 \pm 3.06$ & $6.11 \pm 0.49$ & $15.91 \pm 0.01$ & $0.01811 \pm 0.00030$ & $3.21887 \pm 0.88728$ & $19.491 \pm 0.014$ & 61.58 & $0.03315 \pm 0.00165$ \\
10. & $37.27 \pm 1.94$ & $5.90 \pm 0.31$ & $13.83 \pm 0.02$ & $0.01820 \pm 0.00021$ & $1.98460 \pm 0.60079$ & $16.472 \pm 0.018$ & 57.32 & $0.02683 \pm 0.00104$ \\
11. & $40.65 \pm 2.03$ & $6.45 \pm 0.32$ & $15.35 \pm 0.02$ & $0.01890 \pm 0.00022$ & $1.87399 \pm 0.62420$ & $15.883 \pm 0.014$ & 58.00 & $0.03012 \pm 0.00110$ \\
12. & $41.64 \pm 2.13$ & $6.60 \pm 0.34$ & $15.36 \pm 0.02$ & $0.01851 \pm 0.00023$ & $1.79766 \pm 0.65412$ & $15.167 \pm 0.014$ & 57.00 & $0.02962 \pm 0.00115$ \\
\hline
\end{tabular}

Total gas age $34.56 \pm 4.59 \mathrm{Ma}$ 193.64

Plateau Ar-Ar age 36.92 $\pm 0.46 \mathrm{Ma}$ with $45.5 \%{ }^{39} \mathrm{Ar}$
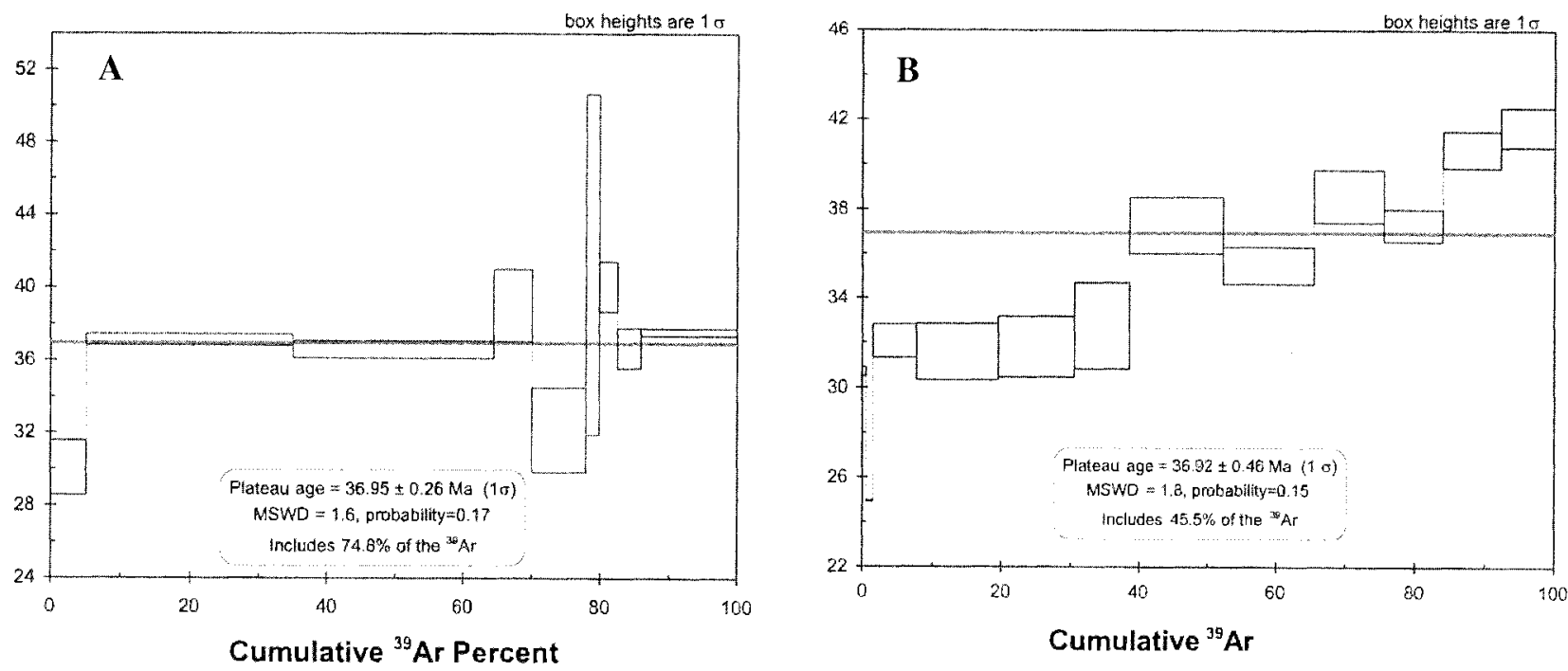

FIG. 2 - Step beating age spectra (apparent age versus cumulative ${ }^{39}$ Ar released). (A) Strathlyn pegmatoidal hawaiite. (B) Cocked Hat Hill hawaiite. 


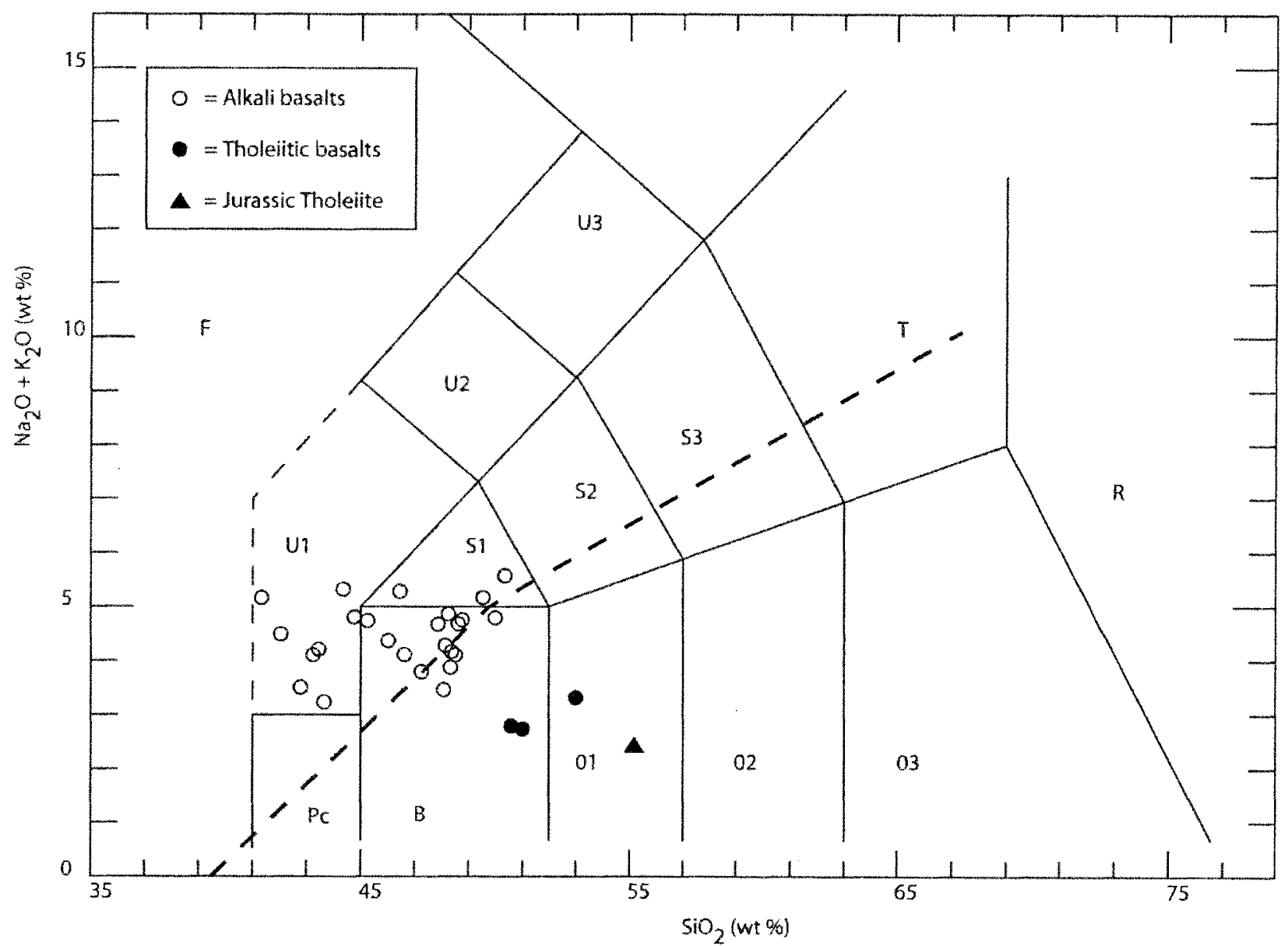

FIG. 3 - Silica against Total Alkalis plots for Tamar basalts related to IUGS basalt categories (Le Maitre 1989) and alkaline and subalkaline fields (Irvine \& Baragar 1971). Occupied fields are: B basalt, U1 basanite, S1 trachybasalt hawaiite, $O 1$ basaltic andesite.

compatible with the underlying Lower-Middle P. tuberculatus Zone (Oligocene) age sediments there and in the Kelso basalt sections to the east. This dates the diversion of the lower Tamar through Kelso as an Oligocene feature. The next group of ages lies between 29-33 Ma (Early Oligocene) from several places along the Tamar Trough. These ages are all consistent with their positions overlying or adjacent to sediments as old as or older than the $N$. asperus Zone (Early Oligocene-Middle Eocene). The higher level hawaiite capping Murphys Hill and the Batman Highway in the Middle Tamar at 32 Ma clearly post-dates earlier flows and Upper $M$. diversus-P. asperopolus Zone (Early-Middle Eocene) age sediments to the west. The Loch Lea basanite near Strathlyn in the Upper Tamar sequence at $34 \mathrm{Ma}$ provides a minimum age for the sediments under the Grïndelwald basalt plateau and is compatible with the $N$. asperus, $M$. diversus and L. balmei Zones (Early OligocenePalaeocene) found in discrete sedimentary sections between Legana and Windermere. The oldest basalt flow dated in the Tamar sections is the Rowella hawaiite at $46.7 \mathrm{Ma}$. This Middle Eocene age is consistent with its interbedded position within Upper $M$. diversus-P. asperopolus Zone (Early-Middle Eocene) sediments and is also compatible with its position below erosional surfaces for further basalt infills extending up sequence from Spring Bay (nephelinite) to the $32 \mathrm{Ma}$ Batman Highway hawaiite. The 'Tamerton' result at $>159$ Ma clearly stands outside the Tamar Trough volcanism as a Jurassic event linked to the extensive dolerite intrusions and did not source any basalt within the Trough.
The follow up Ar-Ar dating on selected basalts (table 2) refines the $\mathrm{K}-\mathrm{Ar}$ dating. This method was attempted on the Rowella hawaiite (K-Ar 46.7 Ma $\pm 1.0 \mathrm{Ma}$ ). The sample, however, only yielded very small amounts of gas, possibly due to contamination. Only five heating steps were possible on each separate split of the sample, which was insufficient to define satisfactory spectra. The best total gas age was $40.06 \pm 1.60 \mathrm{Ma}$ and is best considered a minimum age. The ${ }^{40} \mathrm{Ar} /{ }^{36} \mathrm{Ar}$ intercept in this sample is $165 \pm 21$, which suggests little or no excess Ar is involved. Thus, available data suggests the Rowella hawaiite is $>40 \mathrm{Ma}$ and that a more reliable date is the $\mathrm{K}-\mathrm{Ar}$ age of $46.7 \pm 1.0 \mathrm{Ma}$, which is compatible with the palynological age.

The coarse-grained Grïndelwald plateau hawaiite gave an Ar-Ar Total gas age of $36.7 \pm 3.4 \mathrm{Ma}$ and a plateau age of $37.0 \pm 0.2$ ( 459 , fig. 2 ). Thus, the underlying sediments here are firmly $N$. asperus and older age zones. The age allows for alteration and/or erosion of the Grïndelwald hawaiite prior to contact with Loch Lea basanite as observed at Rosevears Estate. This relationship brackets the Loch Lea basanite to an age between $33.7 \pm 0.7 \mathrm{Ma}(\mathrm{K}-\mathrm{Ar})$ and $37.0 \pm 0.2 \mathrm{Ma}$ (Ar-Ar). It also gives the Tamar drainage a reasonable period to down-cut and re-establish its course through the massive basalts that filled the Early Oligocene channel there. The Cocked Hat Hill hawaiite gave a total gas age of $34.6 \pm 4.6 \mathrm{Ma}$ and a plateau Ar-Ar age of 36.9 $\pm 0.5 \mathrm{Ma}$ (414, fig. 4). The ${ }^{40} \mathrm{Ar} / 36 \mathrm{Ar}$ intercept is $283 \pm 8$, which suggests no excess Ar is present, as it is close to the 
TABLE 3

Whole rock XRF element analyses (wt \%, ppm) and CIPW norms, Tamar Trough basalts

\begin{tabular}{|c|c|c|c|c|c|c|c|c|c|}
\hline Oxide & $\mathrm{BA}^{\prime}$ & $\mathrm{BA}^{2}$ & $\mathrm{BA}^{3}$ & $\mathrm{AB}^{\mathrm{i}}$ & $\mathrm{HA}^{5}$ & $\mathrm{OB}^{6}$ & $\mathrm{OB}^{7}$ & $\mathrm{OT}^{8}$ & $\mathrm{TH}^{9}$ \\
\hline $\mathrm{SiO}_{2}$ & 43.31 & 44.58 & 44.19 & 46.03 & 47.38 & 47.19 & 47.19 & 50.29 & 49.83 \\
\hline $\mathrm{TiO}_{2}$ & 2.76 & 2.43 & 2.52 & 1.95 & 2.05 & 2.06 & 2.31 & 2.01 & 1.73 \\
\hline $\mathrm{Al}_{2} \mathrm{O}_{3}$ & 11.39 & 13.60 & 13.69 & 14.38 & 13.94 & 13.74 & 14.01 & 13.38 & 13.47 \\
\hline $\mathrm{Fe}_{2} \mathrm{O}_{3}$ & 2.51 & 2.40 & 6.22 & 1.68 & 2.61 & 1.37 & 3.60 & 1.66 & 1.16 \\
\hline $\mathrm{FeO}$ & 9.89 & 9.50 & 6.39 & 9.31 & 8.46 & 9.44 & 9.51 & 9.31 & 11.10 \\
\hline $\mathrm{MnO}$ & 0.17 & 0.18 & 0.19 & 0.16 & 0.17 & 0.16 & 0.16 & 0.15 & 0.16 \\
\hline $\mathrm{MgO}$ & 11.49 & 10.86 & 9.70 & 10.17 & 9.43 & 9.84 & 7.80 & 9.14 & 8.27 \\
\hline $\mathrm{CaO}$ & 10.21 & 9.62 & 10.24 & 9.54 & 8.75 & 9.25 & 8.04 & 8.55 & 8.97 \\
\hline $\mathrm{Na}_{2} \mathrm{O}$ & 3.54 & 3.28 & 3.27 & 2.93 & 3.12 & 2.80 & 3.21 & 2.71 & 2.45 \\
\hline $\mathrm{K}_{2} \mathrm{O}$ & 1.22 & 1.35 & 1.47 & 1.27 & 1.11 & 0.86 & 1.04 & 0.73 & 0.30 \\
\hline $\mathrm{P}_{2} \mathrm{O}_{5}$ & 0.75 & 0.53 & 0.74 & 0.44 & 0.51 & 0.48 & 0.45 & 0.33 & 0.26 \\
\hline $\mathrm{H}_{2} \mathrm{O}+$ & 1.60 & 1.54 & 1.23 & 1.60 & 1.86 & 1.82 & 1.80 & 1.11 & 1.22 \\
\hline $\mathrm{CO}_{2}$ & 0.07 & 0.08 & 0.12 & 0.04 & 0.19 & 0.28 & 0.20 & 0.19 & 0.08 \\
\hline $\mathrm{SO}_{3}$ & 0.02 & 0.00 & 0.06 & & 0.11 & 0.02 & 0.07 & 0.05 & 0.11 \\
\hline Total & 98.93 & 99.94 & 100.03 & 99.50 & 99.69 & 99.31 & 99.39 & 99.61 & 99.11 \\
\hline Mg value & 66.55 & 66.20 & 62.98 & 66.40 & 66.35 & 64.75 & 65.97 & 64.04 & 58.89 \\
\hline \multicolumn{10}{|c|}{$\mathrm{CIPW}$ anhydrous $100 \mathrm{wt} \%$ norms, calculated at $\mathrm{Fe}_{2} \mathrm{O}_{3} / \mathrm{FeO}-0.2$} \\
\hline Q & & & & & & & & & 0.82 \\
\hline or & 7.43 & 8.13 & 8.84 & 7.68 & 6.73 & 5.22 & 6.32 & 4.39 & 1.83 \\
\hline$a b$ & 9.35 & 12.64 & 11.39 & 18.38 & 26.92 & 24.36 & 27.92 & 23.31 & 21.23 \\
\hline an & 11.92 & 18.72 & 18.68 & 22.81 & 21.27 & 23.03 & 21.38 & 22.58 & 25.39 \\
\hline ne & 11.62 & 8.45 & 9.08 & 3.77 & 0.12 & & & & \\
\hline di & 28.39 & 21.27 & 22.82 & 18.31 & 16.05 & 16.91 & 13.63 & 14.92 & 14.99 \\
\hline hy & & & & & & 4.78 & 4.66 & 24.87 & 28.68 \\
\hline ol & 21.01 & 21.92 & 19.54 & 21.49 & 20.94 & 17.79 & 17.25 & 2.55 & \\
\hline $\mathrm{mt}$ & 3.07 & 2.91 & 2.99 & 2.71 & 2.72 & 2.69 & 3.21 & 2.70 & 3.04 \\
\hline il & 5.40 & 4.68 & 4.87 & 3.77 & 3.98 & 4.02 & 4.52 & 3.88 & 3.36 \\
\hline ap & 1.81 & 1.22 & 1.71 & 1.01 & 1.20 & 1.13 & 1.04 & 0.79 & 0.61 \\
\hline$A n \%$ & 55.58 & 58.26 & 60.72 & 53.91 & 42.68 & 47.12 & 41.92 & 47.73 & 52.99 \\
\hline D.I. & 28.40 & 29.22 & 29.31 & 29.83 & 33.77 & 29.58 & 34.24 & 27.69 & 23.06 \\
\hline \multicolumn{10}{|c|}{ Trace elements (ppm) } \\
\hline $\mathrm{Ni}$ & 320 & 220 & 210 & 190 & 200 & 200 & 195 & 230 & 250 \\
\hline $\mathrm{Cr}$ & 390 & 290 & 220 & 350 & 290 & 330 & 260 & 340 & 350 \\
\hline Co & 66 & 59 & 49 & 52 & 49 & 52 & 46 & 55 & 47 \\
\hline Sc & 21 & 22 & 20 & 20 & 17 & 21 & 23 & 26 & 25 \\
\hline V & 140 & 140 & 200 & 135 & 175 & 120 & 160 & 130 & 155 \\
\hline $\mathrm{Ba}$ & 280 & 290 & 370 & 250 & 260 & 280 & 200 & 160 & 82 \\
\hline $\mathrm{Rb}$ & 21 & 24 & 30 & 21 & 28 & 16 & 22 & 22 & 8 \\
\hline $\mathrm{Sr}$ & 880 & 770 & 980 & 690 & 1150 & 780 & 490 & 370 & 270 \\
\hline Y & 24 & 21 & 22 & 18 & 20 & 20 & 20 & 24 & 22 \\
\hline$Z \mathrm{r}$ & 250 & 230 & 280 & 185 & 110 & 200 & 210 & 165 & 120 \\
\hline $\mathrm{Nb}$ & 70 & 51 & 65 & 39 & 33 & 41 & 29 & 19 & 11 \\
\hline $\mathrm{La}$ & 38 & 27 & 44 & 25 & 30 & 23 & $<20$ & $<20$ & $<20$ \\
\hline $\mathrm{Ce}$ & 76 & 69 & 100 & 55 & 73 & 63 & 45 & 30 & $<28$ \\
\hline $\mathrm{Nd}$ & 37 & 29 & 43 & 30 & 30 & 29 & 28 & $<20$ & $<20$ \\
\hline $\mathrm{Pb}$ & $<10$ & $<10$ & $<10$ & $<10$ & 15 & $<10$ & 18 & $<10$ & 16 \\
\hline Th & $<10$ & $<10$ & 19 & $<10$ & $<10$ & 15 & $<10$ & $<10$ & $<10$ \\
\hline U & $<10$ & $<10$ & $<10$ & $<10$ & $<10$ & $<10$ & $<10$ & $<10$ & $<10$ \\
\hline $\mathrm{Cu}$ & 51 & 64 & 66 & 53 & 33 & 40 & 53 & 46 & 82 \\
\hline $\mathrm{Zn}$ & 145 & 115 & 130 & 100 & 105 & 120 & 130 & 135 & 120 \\
\hline $\mathrm{Ga}$ & 23 & 22 & 23 & 20 & 22 & 21 & 24 & 22 & 18 \\
\hline$\sum$ Inc. & 797 & 591 & 499 & 612 & 559 & 603 & 524 & 851 & 672 \\
\hline
\end{tabular}

$\Sigma$ Inc. Sum of incompatible elements $\mathrm{Ni}, \mathrm{Cr}, \mathrm{Co}, \mathrm{Sc}$. 
TABLE 3 cont.

\begin{tabular}{lccccccccc}
\hline \multicolumn{2}{l}{ Key element ratios } \\
$\mathrm{Zr} / \mathrm{Nb}$ & 3.57 & 4.51 & 4.31 & 4.74 & 3.33 & 4.88 & 7.24 & 8.68 & 10.91 \\
$\mathrm{La} / \mathrm{Nb}$ & 0.54 & 0.53 & 0.68 & 0.64 & 0.91 & 0.56 & $>0.83$ & $>1.05$ & $>0.40$ \\
$\mathrm{Ba} / \mathrm{Nb}$ & 4.00 & 5.69 & 5.69 & 6.41 & 7.88 & 6.83 & 6.90 & 8.42 & 8.07 \\
$\mathrm{Rb} / \mathrm{Nb}$ & 0.30 & 0.47 & 0.46 & 0.54 & 0.85 & 0.39 & 0.71 & 1.16 & 0.73 \\
$\mathrm{Ba} / \mathrm{La}$ & 7.37 & 10.74 & 8.41 & 10.00 & 8.67 & 12.17 & $>10.00$ & $>5.00$ & $>4.10$ \\
$\mathrm{La} / \mathrm{Y}$ & 1.58 & 1.29 & 2.00 & 1.39 & 1.50 & 1.15 & $<1.00$ & $<0.89$ & $<1.11$ \\
$\mathrm{Ce} / \mathrm{Y}$ & 3.12 & 3.29 & 4.55 & 3.06 & 3.65 & 3.15 & 2.25 & 1.25 & $<1.56$ \\
\hline
\end{tabular}

1 Basanite (BA) Tasman Highway, Distillery Creek (Launceston EQ 212124).

2 Basanite (BA) Rosevears Estate, Strathlyn (Launceston EQ 006233).

3 Basanite (BA) Bridge, Midlands Highway, Perth (Longford EP 152962).

4 Alkali basalt (AB) Rosevears Estate, Strathlyn (Launceston EQ007232).

5 Hawaiite (HA) Cocked Hat Hill, Breadalbane (Longford EQ 1681 0365).

6 Ol-basalt (OB) Bass Highway, Prospect Vale (Launceston EQ 106068).

7 Ol-basalt (OB) Kclso DDH 2/7.0 (Beaconsfield DQ 814491).

8 Ol-tholeiite (OT) Abels Hill Road, St Leonards (Launceston EQ 179113).

9 Tholeiite (TH) Kelso DDH 1/51.6 (Beaconsfield DQ 819489).

TABLE 4

Comparative normalised analyses, hawaiite fractionation, Tamar Trough

\begin{tabular}{|c|c|c|c|c|c|c|c|c|c|c|c|}
\hline \multicolumn{3}{|c|}{ Major \& minor elements } & \multicolumn{3}{|c|}{ CIPW (anhydrous) Norms* } & \multicolumn{3}{|c|}{ Trace elements } & \multicolumn{3}{|c|}{ Rare Earth Elements } \\
\hline Oxide wt $\%$ & ROW & $\mathrm{CHH}$ & wt $\%$ & ROW & $\mathrm{CHH}$ & ppm & ROW & $\mathrm{CHH}$ & ppm & ROW & $\mathrm{CHH}$ \\
\hline $\mathrm{SiO}_{2}$ & 48.27 & 48.61 & or & 6.07 & 6.69 & $\mathrm{Ni}$ & 177 & 53.3 & $\mathrm{La}$ & 22.0 & 22.9 \\
\hline $\mathrm{TiO}_{2}$ & 1.82 & 2.31 & $a b$ & 24.98 & 29.05 & $\mathrm{Cr}$ & 348 & 152 & $\mathrm{Ce}$ & 43.6 & 51.0 \\
\hline $\mathrm{Al}_{2} \mathrm{O}_{3}$ & 14.41 & 16.31 & an & 23.38 & 24.82 & Co & 59 & 48.4 & $\operatorname{Pr}$ & 5.29 & 5.78 \\
\hline $\mathrm{Fe}_{2} \mathrm{O}_{3}{ }^{*}$ & 2.40 & 2.26 & ne & - & 1.37 & $S_{c}$ & 23 & 17.1 & $\mathrm{Nd}$ & 22.6 & 24.4 \\
\hline $\mathrm{FeO}$ & 8.64 & 8.13 & ol & 20.28 & 12.26 & $\mathrm{~V}$ & 203 & 225 & $\mathrm{Sm}$ & 4.71 & 5.17 \\
\hline $\mathrm{MnO}$ & 0.17 & 0.16 & di & 15.28 & 16.77 & $\mathrm{Rb}$ & 16.4 & 11.0 & $\mathrm{Eu}$ & 1.58 & 1.75 \\
\hline $\mathrm{MgO}$ & 10.19 & 6.13 & hy & 2.88 & - & $\mathrm{Sr}$ & 605 & 608 & Gd & 4.74 & 5.05 \\
\hline $\mathrm{CaO}$ & 9.01 & 9.81 & $\mathrm{mt}$ & 2.67 & 3.30 & $\mathrm{Y}$ & 18.7 & 17.9 & $\mathrm{~Tb}$ & 0.67 & 0.72 \\
\hline $\mathrm{Na}_{2} \mathrm{O}$ & 2.93 & 3.70 & i] & 3.48 & 4.42 & $\mathrm{Zr}$ & 141 & 167 & Dy & 3.82 & 4.01 \\
\hline $\mathrm{K}_{2} \mathrm{O}$ & 1.02 & 1.12 & ap & 1.02 & 1.34 & $\mathrm{Nb}$ & 36.4 & 40 & Ho & 0.72 & 0.74 \\
\hline $\mathrm{P}_{2} \mathrm{O}_{5}$ & 0.43 & 0.56 & & & & $\mathrm{Ba}$ & 228 & 219 & Er & 1.87 & 1.89 \\
\hline L.O.I. & 0.76 & 0.90 & & & & $\mathrm{Hf}$ & 2.97 & 3.52 & $\operatorname{Tm}$ & 0.25 & 0.29 \\
\hline Total & 100.05 & 100.00 & & 99.95 & 100.00 & $\mathrm{Ta}$ & 3.28 & 3.30 & $\mathrm{Yb}$ & 1.57 & 1.48 \\
\hline \multirow{2}{*}{$\begin{array}{l}\mathrm{Mg} / \\
\left(\mathrm{Mg}+\mathrm{Fe}^{21}\right) \%\end{array}$} & \multirow{2}{*}{66.5} & \multirow{2}{*}{55.9} & \multirow{2}{*}{$\begin{array}{l}\mathrm{An} / \\
(\mathrm{Ab}+\mathrm{An}) \%\end{array}$} & \multirow{2}{*}{46.8} & \multirow{2}{*}{45.8} & $\mathrm{~Pb}$ & 2.75 & 2.51 & $\mathrm{Lu}$ & 0.23 & 0.21 \\
\hline & & & & & & 'Th & 3.02 & 2.85 & SREE & 114 & 126 \\
\hline \multirow[t]{2}{*}{$\mathrm{Na}_{2} \mathrm{O} / \mathrm{K}_{2} \mathrm{O}$} & \multirow[t]{2}{*}{2.88} & \multirow[t]{2}{*}{3.30} & \multirow{2}{*}{$\begin{array}{l}\text { Eor;ab, } \\
\text { ne (D.I.) }\end{array}$} & \multirow[t]{2}{*}{31.1} & \multirow[t]{2}{*}{36.6} & $U$ & 0.91 & 0.92 & $\mathrm{Cs}$ & 0.8 & 0.35 \\
\hline & & & & & & $\mathrm{Cu}$ & 49.8 & 62.3 & $\sum \mathrm{Ni}, \mathrm{Cr}$ & 525 & 205 \\
\hline
\end{tabular}

${ }^{*}$ Calculated at $\mathrm{Fe}_{2} \mathrm{O}_{3} / \mathrm{FeO}-0.2$

ROW Rowella, DDH2, $114.5 \mathrm{~m}$ depth (Beaconsfield IDQ 930413).

CHH Cocked Hat Hill quarry, $180 \mathrm{~m}$ ASL (Longford EQ 16150330).

Analysts, XRF main elements, M. Loubser; ICPMS trace elements, A. Spath. 


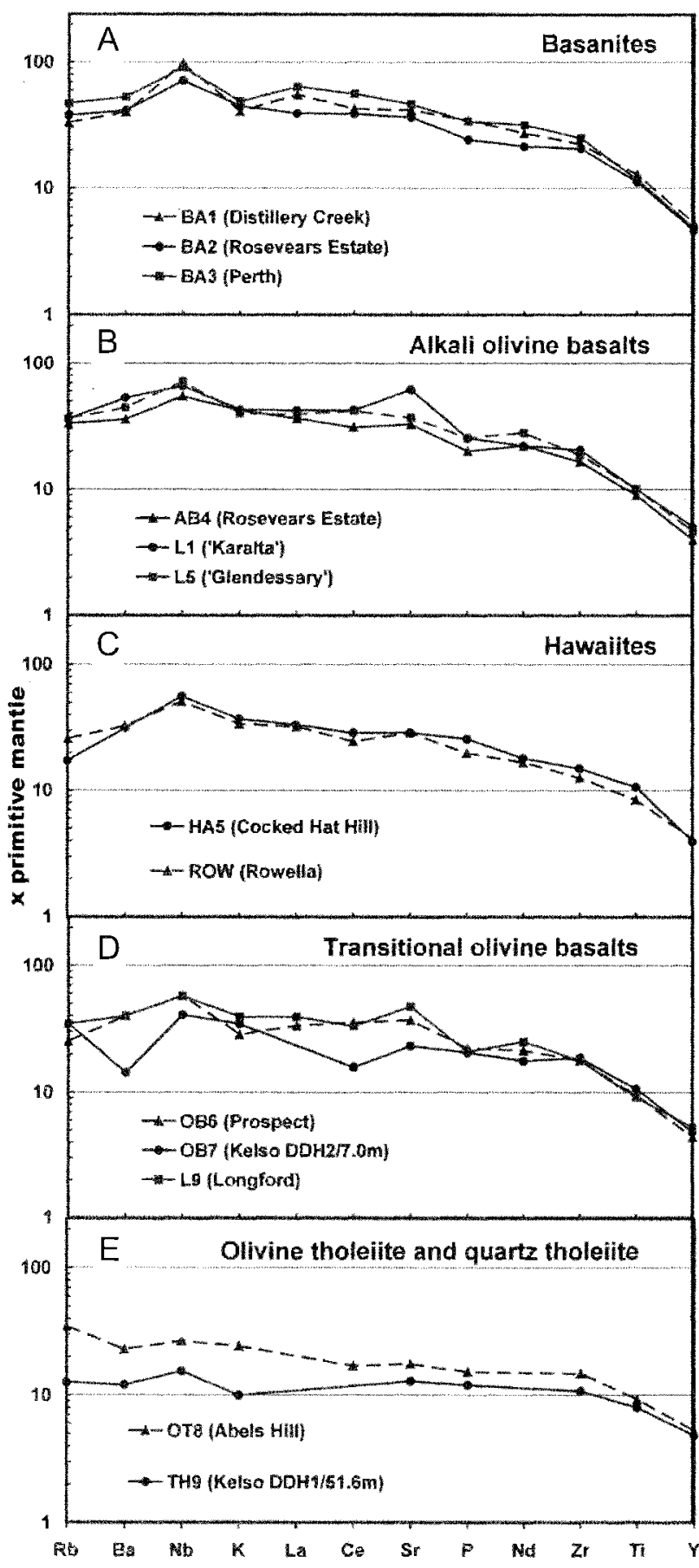

FIG. 4-Primitive Mantle-normalised, multi-element plots, Tamar and Longford basalts (normalising values, after Sun \& McDonough 1989). (A) Basanites, (B) alkali basalts, (C) hawaiites, (D) transitional Ol-basalts, (E) Ol-tholeiite, Qtholeiite.

${ }^{40} \mathrm{Ar} /{ }^{36} \mathrm{Ar}$ air ratios of 295.5. The Ar-Ar age matches the underlying Lower $N$. asperus Zone age and precludes any significant erosion between sediment deposition and hawaiite extrusion. It places the significant westward diversion of the South Esk through Cataract Gorge to the Launceston area to no older than Late Eocene time.

\section{Basalt Geochemistry}

The further basalt analyses (tables 3-5) confirm and expand the previously demonstrated petrological range and include olivine nephelinites and basanites (ne $>5 \%$ ), alkali basalts, hawaiites and transitional olivine basalts (ne $<5 \%$, hy $<10 \%)$ and olivine tholeiites (hy $>10 \%$ ) and quartz tholeiites (hy + Q). The alkaline rocks include near-primary to primary types (Mg values 65-67, An \% 44-81, D.I. 27-34), with compatible element contents ( $\mathrm{Ni}, \mathrm{Cr}, \mathrm{Co}, \mathrm{Sc}$ ) between 525 to $800 \mathrm{ppm}$, and moderately evolved equivalents (Mg values 56-63, An\% 37-61, D.I. 29-38, compatible elements $265-500 \mathrm{ppm}$ ). The olivine and quartz tholeiites show mildly evolved parameters that typify such basalts in Tasmania (Mg values 58-62, An $\%$ 53-54, D.I. 23-24), but retain relatively high compatible element contents (670-850 ppm). The 'Tamerton' qtz tholeiite, now known to be Jurassic in age, is clearly more silicarich (Q 19\%) and calcic (An\% 62) and relatively depleted in compatible elements (204 ppm), compared with the Cenozoic Tamar tholeiites. For total alkalis against $\mathrm{SiO}_{2}$ (fig. 5), the basalts show a general decrease in alkali contents across the alkaline into the subalkaline fields. The bulk of alkali basalts are moderately sodic types $\left(\mathrm{Na}_{2} \mathrm{O} / \mathrm{K}_{2} \mathrm{O} 2.2-3.8\right)$, while the Kelso tholeiites are distinctly low in $\mathrm{K}_{2} \mathrm{O}\left(\mathrm{Na}_{2} \mathrm{O} / \mathrm{K}_{2} \mathrm{O}\right.$ 8.2). A few Tamar alkaline rocks are less sodic in nature, particularly the highly undersaturated rocks in the St Leonards-White Hills area $\left(\mathrm{Na}_{2} \mathrm{O} / \mathrm{K}_{2} \mathrm{O}\right.$ 1.1-1.6; Sutherland 1969).
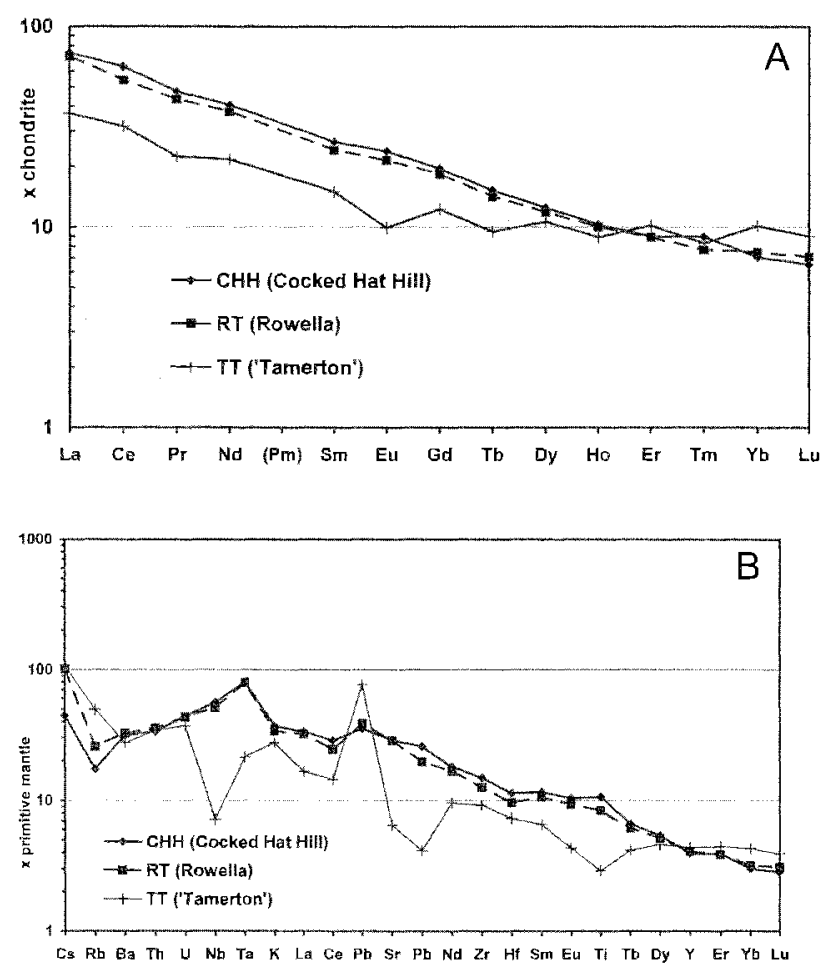

FIG. 5 - Normalised multi-element plots, Tamar alkaline basalts (Cocked Hat Hill, Rowella) and Tamerton quartz tholeiite. (A) REE. (B) Multi-elements. The Tamerton plots utilise unpublished ICPMS analyses (A. Spath, analyst). Chondrite normalising values are taken from Boynton (1984) and Primitive Mantle normalising values are after Sun do McDonough (1989). 
TABLE 5

Comparative analyses and CIPW Norms, tholeiites, Tamar Trough

\begin{tabular}{|c|c|c|c|c|c|c|c|c|c|c|c|}
\hline \multicolumn{3}{|c|}{$\begin{array}{l}\text { Major and minor elements } \\
\text { (wt \%) }\end{array}$} & \multicolumn{3}{|c|}{$\begin{array}{c}\text { CIPW (anhydrous) Norms* } \\
\text { (wt } \%)\end{array}$} & \multicolumn{3}{|c|}{ Trace elements (ppm) } & \multicolumn{3}{|c|}{ Rare Earth Elements (ppm) } \\
\hline Oxide & $\mathrm{TT}$ & $\mathrm{KT}$ & & $\mathrm{TT}$ & $\mathrm{KT}$ & & $\mathrm{TT}$ & $\mathrm{KT}$ & & $\mathrm{TT}$ & $\mathrm{KT}$ \\
\hline $\mathrm{SiO}_{2}$ & 55.21 & 49.52 & $Q$ & 10.08 & 0.05 & $\mathrm{Ni}$ & 76 & 220 & $\mathrm{Rb}$ & 35 & 7 \\
\hline $\mathrm{TiO}_{2}$ & 0.63 & 1.78 & or & 4.91 & 1.83 & $\mathrm{Cr}$ & 41 & 370 & $\mathrm{Sr}$ & 122 & 270 \\
\hline $\mathrm{Al}_{2} \mathrm{O}_{3}$ & 14.75 & 13.94 & $a b$ & 13.56 & 21.32 & Co & 63 & 48 & $\mathrm{Y}$ & 23 & 23 \\
\hline $\mathrm{Fe}_{2} \mathrm{O}_{3}$ & 1.46 & 1.77 & an & 30.59 & 26.71 & $\mathrm{Sc}$ & 24 & 30 & $\mathrm{Zr}$ & 103 & 120 \\
\hline $\mathrm{FeO}$ & 7.32 & 9.51 & $\mathrm{di}$ & 16.85 & 15.44 & V & 230 & 170 & $\mathrm{Nb}$ & 7 & 11 \\
\hline $\mathrm{MnO}$ & 0.19 & 0.17 & hy & 20.42 & 27.63 & $\mathrm{Cu}$ & 82 & 53 & $\mathrm{Ba}$ & 215 & 80 \\
\hline $\mathrm{MgO}$ & 6.69 & 8.54 & $\mathrm{mt}$ & 2.13 & 2.79 & $\mathrm{Zn}$ & 71 & 115 & $\mathrm{La}$ & 46 & $<20$ \\
\hline $\mathrm{CaO}$ & 10.82 & 9.41 & il & 1.20 & 3.45 & $\mathrm{~Pb}$ & 9 & 11 & $\mathrm{Ce}$ & 29 & $<28$ \\
\hline $\mathrm{Na}_{2} \mathrm{O}$ & 1.60 & 2.46 & ap & 0.21 & 0.72 & $\mathrm{Ga}$ & 16 & 20 & $\mathrm{Nd}$ & - & $<20$ \\
\hline $\mathrm{K}_{2} \mathrm{O}$ & 0.83 & 0.30 & Total & 99.95 & 99.94 & The & 3 & $<10$ & & & \\
\hline $\mathrm{P}_{2} \mathrm{O}_{5}$ & 0.09 & 0.31 & $\mathrm{An} /$ & & & U & 3 & $<10$ & & & \\
\hline Total & 99.74 & 98.71 & $(A b+A n) \%$ & 69.3 & 54.1 & $\sum \mathrm{Ni}, \mathrm{Cr}$ & 117 & 590 & $\sum \mathrm{La}, \mathrm{Ce} 75$ & $<48$ & \\
\hline $\mathrm{Mg} \#$ & 62.02 & 61.8 & $\sum \mathrm{Q}, \mathrm{or}, \mathrm{ab}$ & & & & & & & & \\
\hline $\mathrm{Na}_{2} \mathrm{OK}_{2} \mathrm{O}$ & 1.93 & 8.2 & (D.I.) & 28.6 & 23.2 & & & & & & \\
\hline
\end{tabular}

$\mathrm{Mg}$ value and CIPW norms calculated at $\mathrm{Fe}_{2} \mathrm{O}_{3} /(\mathrm{FeO})$ 0.2. TT "Tamerton" basaltic plug (Frankford DQ 4920000 54302000). Analyst, XRF analyses, M. Loubser. KT Kelso DDH 2/27.5 m tholeïte flow (Beaconsfield DQ 4814000 54491000). Analyst, XRF analyses, Tasmanian Mineral Resources Laboratory.

TABLE 6

Most mafic, least evolved basalts, Tamar Trough

\begin{tabular}{|c|c|c|c|c|}
\hline $\begin{array}{l}\text { Basalt Type } \\
\text { (criteria) }\end{array}$ & $\begin{array}{c}\text { Ol.nephelinite } \\
(\mathrm{ab}<5 \%, \text { ne }>5 \%)\end{array}$ & $\begin{array}{c}\text { Basanite } \\
(a b>5 \%, a b<a n)\end{array}$ & $\begin{array}{c}\text { Alkali basalt-hawaiite } \\
\text { (ne,hy<5\%, ab<an) }\end{array}$ & $\begin{array}{l}\text { Tholeiitic basalt } \\
\text { (hy> } 10 \%, \pm Q \text { ) }\end{array}$ \\
\hline \multicolumn{5}{|c|}{ CIPW norms (wt \%)* } \\
\hline $\mathrm{Q}$ & - & - & - & $0.1-0.8$ \\
\hline or & 9.2 & $6.3-8.9$ & $6.1-7.9$ & $1.8-1.8$ \\
\hline$a b$ & 3.0 & $7.5-12.6$ & $18.4-24.9$ & $21.2-21.3$ \\
\hline an & 13.1 & $18.7-21.5$ & $22.8-23.6$ & $25.49-26.7$ \\
\hline ne & 15.5 & $8.4-9.9$ & $0.0-0.4$ & - \\
\hline ol & 25.3 & $19.5-21.9$ & $17.8-22.9$ & - \\
\hline di & 21.9 & $21.3-23.2$ & $15.4-18.3$ & $15.0-15.4$ \\
\hline hy & - & - & $0.0-4.8$ & $27.6-28.7$ \\
\hline $\mathrm{mt}$ & 3.7 & $2.9-3.3$ & $2.7-3.5$ & $2.8-3.0$ \\
\hline il & 5.5 & $4.7-4.9$ & $3.8-4.0$ & $3.4-3.6$ \\
\hline ap & 2.9 & $1.2-1.9$ & $1.0-1.2$ & $0.6-0.7$ \\
\hline \multicolumn{5}{|c|}{ Fractionation indices } \\
\hline $\mathrm{Mg} /\left(\mathrm{Mg}+\mathrm{Fe}^{2+}\right)^{*} \%$ & 62.1 & $62.3-66.2$ & $64.8-67.9$ & $58.9-61.8$ \\
\hline$A n /(A b+A n) \%$ & 81.6 & $58.3-74.1$ & $47.1-53.9$ & $53.0-54.1$ \\
\hline$\sum$ or, ab, ne (D.I.) & 27.6 & $23.7-29.3$ & $29.6-33.8$ & $23.1-23.2$ \\
\hline \multicolumn{5}{|c|}{ Key compatible elements } \\
\hline $\mathrm{Ni}$ & 305 & $210-220$ & $175-200$ & $200-250$ \\
\hline $\mathrm{Cr}$ & 341 & $220-290$ & $278-420$ & $350-370$ \\
\hline V & 173 & $140-200$ & $120-240$ & $155-170$ \\
\hline \multicolumn{5}{|c|}{ Key incompatible elements } \\
\hline $\mathrm{Rb}$ & 20 & $24-30$ & $16-28$ & $7-22$ \\
\hline $\mathrm{Sr}$ & 975 & $770-980$ & $522-1150$ & $270-370$ \\
\hline $\mathrm{Ba}$ & 371 & $290-370$ & $185-280$ & $84-120$ \\
\hline Y & 34 & $21-29$ & $18-26$ & $22-240$ \\
\hline $\mathrm{Zr}$ & 323 & $230-285$ & $110-200$ & $120-165$ \\
\hline
\end{tabular}

Data from analyses in Sutherland (1969) and F.L. Sutherland unpublished trace element analyses and from Tables 3-5 this paper.

* Calculated at $\mathrm{Fe}_{2} \mathrm{O}_{3} / \mathrm{FeO} \sim 0.2$. 
TABLE 7

Late fractionates, Tamar basalts

\begin{tabular}{|c|c|c|}
\hline Pegmatoid type & In hawaiite & In basanite \\
\hline \multicolumn{3}{|c|}{ CIPW norms (anhydrous wt \%) } \\
\hline or & 9.3 & 6.7 \\
\hline $\mathrm{ab}$ & 34.7 & 28.0 \\
\hline an & 16.5 & 17.7 \\
\hline ne & - & 1.7 \\
\hline $\mathrm{ol}$ & 9.6 & 20.4 \\
\hline $\mathrm{di}$ & 17.6 & 16.5 \\
\hline hy & 0.4 & - \\
\hline $\mathrm{mt}$ & 4.2 & 3.2 \\
\hline il & 6.2 & 4.6 \\
\hline ap & 1.5 & 1.0 \\
\hline \multicolumn{3}{|c|}{ Fractionation indices } \\
\hline $\mathrm{Mg} /\left(\mathrm{Mg}+\mathrm{Fe}^{2+}\right) \%$ & 51.7 & 59.9 \\
\hline$A n /(A b+A n) \%$ & 32.2 & 38.7 \\
\hline$\sum$ or,ab,ne (D.I.) & 44.0 & 29.8 \\
\hline \multicolumn{3}{|c|}{ Key compatible elements } \\
\hline $\mathrm{Ni}$ & 49 & \\
\hline $\mathrm{Cr}$ & 42 & \\
\hline V & 217 & \\
\hline \multicolumn{3}{|c|}{ Key incompatible elements } \\
\hline $\mathrm{Rb}$ & 33 & \\
\hline Sr & 535 & \\
\hline $\mathrm{Ba}$ & 159 & \\
\hline Y & 33 & \\
\hline $\mathrm{Zr}$ & 231 & \\
\hline
\end{tabular}

Data from analyses in Sutherland (1969) and F.L. Sutherland unpublished trace element analyses.

CIPW norms and $\mathrm{Mg}$ values calculated at analysed $\mathrm{Fe}_{2} \mathrm{O}_{3} / \mathrm{FeO}$ ratios.

The expanded range of Tamar basalt geochemistry (Sutherland 1969 and this paper) mostly includes near primary to mildly evolved magmas (tables $4,6,8$ ), but some of the more voluminous flows (Craigburn-East Arm, parts of Grindelwald plateau, Cocked Hat Hill - South Esk) represent more fractionated magmas, further depleted in compatible elements and enriched in incompatible elements (see table 4 for comparison). Limited post-eruptive differentiation occurs in some basalts, where pegmatoidal developments formed during prolonged cooling and volatile build-up; these later stage phases show increased depletions in compatible elements $(\mathrm{Ni}, \mathrm{Cr}<100 \mathrm{ppm})$ and greater enrichments in Zr, Rb, Y (table 7).

Key incompatible element ratios show distinct differences across the basalt types (tables $3 \& 8$ ). The alkaline basalts, relative to the subalkaline basalts, have lower $\mathrm{Zr} / \mathrm{Nb}(3-6$ of 7-11) and higher $\mathrm{Ba} / \mathrm{La}(8-35$ of $4-5), \mathrm{La} / \mathrm{Y}(1-2$ of 1) and $\mathrm{Ce} / \mathrm{Y}(2-5$ of $1-2)$ ratios. These differences relate to different degrees of partial mantle melting within the mantle sources that increasingly depleted various mineral phases. Irregular variations in some values, however, such as $\mathrm{Ba}$ contents and $\mathrm{Ba} / \mathrm{La}$ and $\mathrm{Ba} / \mathrm{Nb}$ ratios suggest some specific phase differences also exist within the sources. The Cocked Hat Hill hawaiite flow has the lowest $\mathrm{Zr} / \mathrm{Nb}$ (3.3) and highest $\mathrm{La} / \mathrm{Nb}(0.9)$ and $\mathrm{Rb} / \mathrm{Nb}(0.9)$ ratios even when compared to its more evolved plug composition $(\mathrm{Zr} / \mathrm{Nb}$ 3.9, $\mathrm{La} / \mathrm{Nb} 0.6, \mathrm{Rb} / \mathrm{Nb} 0.5$ ). Thus, some key element ratios changed perceptibly during fractionation even within a How episode.

\section{DISCUSSION}

Correlations of the basalt dating and known palynological biozonation within the associated Tamar sequences are given in table 9. This forms a new, more rigorous framework for the sedimentological, erosive and eruptive history within the Tamar Trough since its inception.

\section{Basalt Geochronology}

The K-Ar and Ar-Ar dating identifies several separate volcanic episodes in the evolution of the Tamar Trough; these include three prominent volcanic intervals that extend from Middle Eocene (47-46 Ma) through Late Eocene (37-32 Ma) to Late Oligocene (25-24 Ma) time. Differences between K-Ar and Ar-Ar results on the same basalt can range up to 8 million years (Rowella and Cocked Hat Hill hawaiites), giving some limits for uncertainties in the dating. Discrepancies between the two dating techniques may be due to Ar loss from late-stage alteration (Cocked Hat Hill, $29 \mathrm{~K}-\mathrm{Ar}$ of $37 \mathrm{Ar}-\mathrm{Ar} \mathrm{Ma}$ ), and lor from contaminated degassing (Rowella, $40 \mathrm{Ar}-\mathrm{Ar}$ if 47 $\mathrm{K}$-Ar Ma). In such cases it was important to evaluate excess argon effects in the K-Ar dating material in the K-Ar ages, and this was done by inspection of Ar-Ar data. Even with dating discrepancies, likely basalt ages can be constrained through the associated palynological biozone ages, to indicate the more reliable date. Thus, the congruence of palynological age (Middle N. asperus) and Ar-Ar basalt age (37 Ma) at Cocked Hat Hill and also the matching palynological age (Upper $M$. diversus or P. asperopolus Zone) and K-Ar basalt age ( $47 \mathrm{Ma}$ ) at Rowella help confirm these radiometric dates.

The oldest Tamar basalt in the Middle Tamar channel through Rowella at 47 Ma ranks among the older Tasmanian eruptives, although this age is exceeded only by basalts on the eastern Tasman margin (Cape Portland, Bream Creek) and on King Island (59-70 Ma; Baillie 1987, Sutherland et al. 2002, Everard et al. 2004a, F.L. Sutherland and H. Zwingmann, unpublished data). The Rowella episode correlates with and extends the alkali basaltic activity known around Weldborough, NE Tasmania (46-47 Ma; Sutherland \& Wellman 1986).

The most voluminous basaltic infillings were produced by the Late Eocene eruptives (37-33 Ma) in the Middle and South Tamar areas. Two major flows (Rosevears, Bredalbane) have Ar-Ar ages within experimental error (37 Ma), and with a further voluminous flow at East Arm and other flows in the Middle to South Tamar region (> 32-37 Ma), mark a peak in volcanism within the Tamar Trough. These massive outpourings caused considerable dislocations to Tamar courses including westward diversion of the South Esk from ancestral Tamar drainage. Basalts in the 33-38 Ma bracket also prevail elsewhere in Tasmania (Blessington, Thirlstane, Lillicos Beach, Melton Mowbray, Central plateau; Cromer 1980, Sutherland \& Wellman 1986, Everard et al. 2004b). Indeed they may include extensive basalts through NW Tasmania, east of Waratah where interbedded sediments include the Upper $N$. asperus and Lower P. tuberculatus biozones (Brown \& Forsyth 1984). Nevertheless, the Late 
TABLE 8

Comparative key trace element ratios, Tamar and Tasmanian basalts

\begin{tabular}{|c|c|c|c|c|c|c|c|c|c|}
\hline Basalt/key element ratios & Mg value & $\mathrm{Zr} / \mathrm{Nb}$ & $\mathrm{La} / \mathrm{Nb}$ & $\mathrm{Ba} / \mathrm{Nb}$ & $\mathrm{Rb} / \mathrm{Nb}$ & $\mathrm{Ba} / \mathrm{Th}$ & $\mathrm{Ba} / \mathrm{La}$ & $\mathbf{L a} / \mathbf{Y}$ & $\mathrm{Ce} / \mathrm{Y}$ \\
\hline Tamar 'mafic' basanite' & 66 & 4.51 & 0.53 & 5.69 & 0.47 & $>29$ & 10.7 & 1.29 & 3.29 \\
\hline Tamar 'mafic' alk basalt ${ }^{2}$ & 66 & 4.74 & 0.64 & 6.41 & 0.54 & $>25$ & 10.0 & 1.39 & 3.06 \\
\hline Tamar 'mafic' hawaiite. & 65 & 3.33 & 0.91 & 7.88 & 0.85 & $>26$ & 8.67 & 1.50 & 3.65 \\
\hline Tamar 'mafic' ol basalt $t^{4}$ & 66 & 4.88 & 0.56 & 6.83 & 0.39 & 18.7 & 12.2 & $<5.0$ & 3.15 \\
\hline Tamar primary hawaiite 5 & 68 & 3.87 & 0.60 & 6.26 & 0.45 & 75.5 & 10.4 & 1.18 & 2.33 \\
\hline Tamar evolved hawaiite ${ }^{6}$ & 61 & 4.18 & 0.57 & 5.48 & 0.28 & 76.8 & 9.56 & 1.28 & 2.85 \\
\hline Longford alkali basalts av.? & 65 & 4.63 & 0.13 & 6.93 & 0.50 & - & 11.6 & 1.20 & 3.10 \\
\hline NE Tas alkali basalt ${ }^{8}$ & 61 & 3.62 & 0.54 & 5.19 & 0.37 & 82.3 & 9.69 & 0.90 & 1.94 \\
\hline NE Tas evolved hawaiite ${ }^{9}$ & 59 & 4.86 & 0.79 & 5.86 & 0.52 & 76.8 & 7.46 & 1.94 & 3.96 \\
\hline NW Tas basanite ${ }^{10}$ & 62 & 3.39 & 0.62 & 6.74 & 0.68 & 86.3 & 10.9 & 1.81 & 3.28 \\
\hline Tasmanian seamounts ${ }^{11}$ & 67 & $3-5$ & $0.5-1$ & $5-8$ & $0.3-1$ & $64-99$ & $9-13$ & $0.9-1.9$ & $2-3.8$ \\
\hline Tamar Q tholeiite ${ }^{12}$ & 59 & 10.9 & $>0.4$ & 8.07 & 0.73 & - & $>4.1$ & $<1.11$ & $<1.56$ \\
\hline NE Tas Q tholeiite $^{13}$ & 63 & 8.05 & 0.56 & 7.85 & 0.63 & 85.0 & 14.0 & 0.41 & 1.50 \\
\hline Loira Q tholeiite ${ }^{14}$ & 62 & 20.0 & 2.22 & 37.4 & 6.11 & 66.2 & 16.8 & 0.57 & 1.29 \\
\hline Tasmanian Q dolerite ${ }^{15}$ & 60 & 21.1 & 2.42 & 48.2 & 7.33 & 62.0 & 19.9 & 0.55 & 1.22 \\
\hline
\end{tabular}

${ }^{1}$ Strathlyn (table 3). ${ }^{2}$ Strathlyn (table 3). ${ }^{3}$ Cocked Hat Hill (table 3). ${ }^{4}$ Prospect Vale (table 3). ${ }^{5}$ Rowella DDH2 (table 4). ${ }^{6}$ Cocked Hat Hill quarry (table 4). ${ }^{7}$ Longford alkaline basalts (this paper, Appendix B). ${ }^{8}$ Weldborough Pass, Blue Tier (Sutherland et al. 2004). ${ }^{9}$ George Rocks dyke (A. Sparh analyst). ${ }^{10}$ Currie, King Island, plug (Sutherland et al. 2004). ${ }^{11}$ Balleny plume trace (Lanyon 1994). ${ }^{12} \mathrm{Kelso}$ DDH 1/ 51.6 (table 3). ${ }^{13}$ North Pipers Brook (Sutherland et al. 2004). ${ }^{14}$ "Tamerton", Supply Creek, Loira (A. Spath analyst). ${ }^{15}$ Tasmanian chilled Jurassic dolerite average (Hergt et al. 1989).

TABLE 9

Summary of sedimentation/volcanism infill sequences, Tamar Trough

\begin{tabular}{|c|c|c|c|c|c|c|}
\hline Age (Ma) & Epoch & Palynology biozone & Date & Basalt type & Area & Comments \\
\hline \multirow[t]{2}{*}{$0-22.5$} & $\begin{array}{l}\text { Holocene- } \\
\text { E. Miocene }\end{array}$ & $\begin{array}{l}\text { 'T. pleistocenicus- } \\
\text { U. P. tuberculatus' }\end{array}$ & & $\begin{array}{l}\text { No definite } \\
\text { records }\end{array}$ & Tamar Valley & $\begin{array}{l}\text { Tamar, river erosion, } \\
\text { sediments and soils }\end{array}$ \\
\hline & E. Miocene? & U. P. tuberculatus? & $?$ & ol.tholeiite? & S. Tamar & Sub-basalt deposits \\
\hline $23.5-32.5$ & $\begin{array}{l}\text { E. Miocene- } \\
\text { E. Oligocene }\end{array}$ & $\begin{array}{l}\text { U. P. tuberculatus? } \\
\text { M. P. tuberculatus- } \\
\text { L. P. tuberculatus }\end{array}$ & $\begin{array}{l}? \\
? \\
24.7^{+}\end{array}$ & $\begin{array}{l}\text { ol.basalt? } \\
\text { hawaiite } \\
\text { tholeiite }\end{array}$ & L-M Tamar & $\begin{array}{l}\text { Shift of Tamar outlet, fluvial } \\
\text { beds, erosion, lava infills, } \\
\text { fuvial beds (dolerite gravels) }\end{array}$ \\
\hline & E. Oligocene? & & & & & \\
\hline $32.5-37.0$ & L. Eocene & $\begin{array}{l}\text { U. N. asperus- } \\
\text { L. N. asperus }\end{array}$ & $\begin{array}{l}32.5^{*} \\
33.7^{*} \\
36.9^{\star} \\
37.0^{2}\end{array}$ & $\begin{array}{l}\text { hawaiite } \\
\text { basanite } \\
\text { hawaiite } \\
\text { hawaiite }\end{array}$ & $\begin{array}{l}\text { M-S Tamar } \\
\text { U. Tamar } \\
\text { S. Tamar }\end{array}$ & $\begin{array}{l}\text { Diversions of Tamar } \\
\text { courses, erosion and } \\
\text { fillings. Diversion of } \\
\text { S. Esk from Tamar }\end{array}$ \\
\hline $37.0-47.0$ & M. Eocene & $\begin{array}{l}\text { L. N. asperus- } \\
\text { P. asperopolus }\end{array}$ & $\begin{array}{l}? \\
? \\
46.7^{+}\end{array}$ & $\begin{array}{l}\text { basanite } \\
\text { nephelinites } \\
\text { hawaiite }\end{array}$ & $\begin{array}{l}\text { M-S Tamar } \\
\text { M. Tamar }\end{array}$ & $\begin{array}{l}\text { Erosion, sediments, } \\
\text { diversions of Tamar, } \\
\text { lava infills }\end{array}$ \\
\hline $47.0-55.0$ & $\begin{array}{l}\text { M. Eocene- } \\
\text { E. Eocene }\end{array}$ & $\begin{array}{l}\text { P. asperopolus- } \\
\text { U. M. diversus }\end{array}$ & & & M-U Tamar & $\begin{array}{l}\text { Trough erosion, fluvial } \\
\text { deposits }\end{array}$ \\
\hline $55.0-65.0$ & Palaeocene & $\begin{array}{l}\text { M-L } M . \text { diversus- } \\
\text { L. balmei- }\end{array}$ & & & Tamar Trough & $\begin{array}{l}\text { Infill sedimentation growth } \\
\text { faulting }\end{array}$ \\
\hline $65.0-95$ & $\begin{array}{l}\text { L. Cretaceous- } \\
\text { M. Cretaceous }\end{array}$ & $F$ longus & & & $\begin{array}{l}\text { TFZ } \\
\text { TM }\end{array}$ & $\begin{array}{l}\text { Main faulting, uplift } \\
\text { Thermal upwelling }\end{array}$ \\
\hline
\end{tabular}

Epoch: L. Late, M. Middle, E. Early; Biozones U. Upper, M. Middle, L. Lower; from Glenn et al. (1999).

+ $\mathrm{K}$-Ar date, * minimum $\mathrm{K}$-Ar date, ${ }^{2} \mathrm{Ar}-\mathrm{Ar}$ date.

Tamar areas: L. Lower, M. Middle, U. Upper, S. South. TFZ Tamar Fracture Zone; TM Tasman Margin. 
Eocene Tamar basalts clearly form a major contribution to Tasmanian volcanism in this period.

The younger basaltic infillings in the Tamar Trough include the more confined Lower Tamar flows. Here, basal tholeiites are interbedded with Lower $P$. tuberculatus zone age beds and lie below hawaiite and transitional olivine basalt flows that overlie Lower-Middle $P$. tuberculatus age sedimentary beds dated between 24-25 Ma. Basalts with 20-25 Ma ages are particularly prevalent throughout the Tasmanian province (Sutherland \& Wellman 1986, Sutherland et al. 2002, 2004, Everard et al. 2004b). Tholeiitic basalts are prominent and the Tamar Trough appears no exception, particularly if the olivine tholeiite in the South Tamar also fits here. So far, no later volcanism is known in the Tamar Trough to match the last phase of alkaline activity in northern Tasmania (9-20 Ma; McClenaghan et al. 1982, Baillie 1986, Sutherland et al. 1996, 2002, Everard et al. 2004b).

\section{Palynology Zones}

Many Tasmanian palaeobotanical sites have been studied, but only a few of the inland sites have radiometric basalt dates directly or indirectly associated with them (Wilmot Dam, Monpeelyata, Lemonthyme). Some of the most important sites lack such dates (Little Rapid River, Lea River), but are associated with basalts (Jordan \& Hill 2002). The Tamar basalt dates provide new control points for palynology zones identified in accompanying sediments. Thus, the $37 \mathrm{Ma} \mathrm{Ar-}$ Ar age at Cocked Hat Hill provides constraints for the top of the Lower $N$. asperus zone which underlies this basalt, and only allows a limited erosive interval here before the Middle $N$. asperus zone (Glenn et al. 1999). Similarly, the $47 \mathrm{Ma}$ $\mathrm{K}$-Ar age at Rowella provides a peg within undifferentiated $P$. asperopolus- $M$. diversus Zone age sediments and suggests they belong to the $P$. asperopolus Zone on the Glenn et al. (1999) correlations. The $25 \mathrm{Ma}$ date on the lower basalt at George Town would reposition the underlying L.-M. P. tuberculatus Zone correlation into the M. P. tuberculatus zone in the Glenn et al. (1999) correlation. The other basalt dates represent minimum ages, so are less precise for pegging biozonation ages. However, minimum dates of $32.5 \mathrm{Ma}$ at Batman Highway, East Arm and $33.7 \mathrm{Ma}$ at Loch Lea, Strathlyn are compatible with pre-basalt erosion into $N$. asperus age sediments.

The correlation of the palynology zones and absolute time scale is dependent on the time scale chosen for the correlations. Thus earlier correlations of basalts and palynology zones in Tasmania (Seymour 1989), differ from those given in this study, which are based on the Glenn et al. (1999) correlations. Uncertainties in previous Tasmanian palynological time correlations, which lacked direct dating, due to use of zones from outside Tasmania and to interpolations in the geomagnetic time scale, were discussed in Seymour (1989). Thus, a basalt age of $47 \mathrm{Ma}$ (Rowella) in earlier correlations would lie at the base of Lower $N$. asperus zone and not within the $P$. asperopolus zone as given in this study. Similarly, basalt ages of $37 \mathrm{Ma}$ (Grïndelwald, Cocked Hat Hill) would lie in the Upper $N$. asperus zone, not in the Middle $N$. asperus zone and the 25 Ma basalt age (George Town) suggests underlying beds are either Middle or Lower $P$. tuberculatus, zone age. The new Tamar study, thus, provides a firmer framework for Tasmania Early Eocene to Early Miocene palynological correlations.

\section{Basalt petrogenesis}

The Tamar basalts represent a range of alkaline to subalkaline magma compositions (fig. 3), but they lack the more undersaturated melilite-bearing types and the more evolved alkaline types found elsewhere in Tasmania (Sutherland et al. 2004, Zhang et al. 2006). The primary magma types identified in the Tasmanian basalt spectrum have been linked to different degrees of partial melting of mantle sources, initially estimated by portioning $\mathrm{K}_{2} \mathrm{O}$ and $\mathrm{P}_{2} \mathrm{O}_{5}$ from the source into the melt (Frey et al. 1978, McClenaghan et al. 1982, Everard in Seymour 1989). The Tamar petrological range (olivine nephelinite to quartz tholeiite) would represent a range from 7 to $35 \%$ in degrees of partial mantle melting using that method. Uncertainties in precise elemental enrichments and melting processes within mantle sources, however, make such estimates very approximate. Thus, trace element inversion modelling on basalts with similar major element compositions to Tasmanian basalts can provide different sets of partial mantle melting degrees. Examples include 3-30\% (Iblean basalts, Sicily; Beccaluva et al. 1998), 2-7\% (Vogelsberg basalts, Germany; Jung \& Marsberg 1998) and $4-11 \%$ (Nushan and Fangshan basalts, China; Zou et al. 2000). Comparison of these trace element contents with those of the Tamar basalts suggest the latter may have been produced by $>3-4 \%$ degrees of partial mantle melting (olivine nephelinite), > 4-6\% (basanites), > 6-10\% (alkali basaltshawaiites), $>9-15 \%$ (olivine tholeiite) and $>16-30 \%$ (quartz tholeiite). New studies show that many Tasmanian basalts include a deep seated metasomatic component to account for their enriched elements (Adam \& Green 2006).

\section{Trace element arrays}

Trace element patterns, normalised to primitive mantle or chondrite values, show variations between the basalt types (figs 4, 5). Basanites and some alkali basalts and hawaiites show relatively subdued $\mathrm{Rb}, \mathrm{Ba}, \mathrm{La}, \mathrm{Ce}$ and $\mathrm{Sr}$ in their profiles, but several have marked enrichments in $\mathrm{Nb}$ and relatively minor depletions in $\mathrm{K}, \mathrm{Ti}$ and $\mathrm{Y}$ (fig. 4A-C). Among hynormative alkali and transitional olivine basalts (fig. 4C), a Kelso olivine basalt is noticeably depleted in $\mathrm{Ba}, \mathrm{La}, \mathrm{Ce}$ and $\mathrm{Sr}$; such depletions approach those shown by the Kelso quartz tholeiites (fig. 4D) and suggest a transitional tholeiitic affinity. The Kelso quartz tholeiites lack the positive $\mathrm{Nb}$ and negative $\mathrm{K}$ anomalies found in the more alkaline basalt patterns and show depletions in $\mathrm{Rb}$ and $\mathrm{Sr}$; the patterns are quite distinct from the Tamerton quartz tholeiite pattern, which shows a classic Jurassic chilled dolerite pattern (Hergt et al. 1989), with strong depletions in $\mathrm{Nb}, \mathrm{Sr}$ and $\mathrm{Ti}$ and significantly higher $\mathrm{Rb}, \mathrm{Ba}$, Th and $\mathrm{K}$ (figs $4 \mathrm{C}, 5$ ).

More extended, primitive mantle-normalised, multielement arrays are available for two prominent Tamar alkali basalts, the Rowella and Cocked Hat Hill basalts (fig. 5). Both show similar normalised REE arrays, that regularly decrease from LREE through MREE to HREE, and suggest magmas derived from a LREE-enriched source (fig. $5 \mathrm{~A}$ ). The extended primitive mantle-normalised patterns are marked by moderate depletions in $\mathrm{Rb}, \mathrm{K}$ and $\mathrm{Hf}$ and enrichments in $\mathrm{Cs}, \mathrm{Nb}$, and $\mathrm{Zr}$ (fig. 5B, C). The fractionated Cocked Hat Hill hawaiite lacks any Eu anomaly, while noticeable depletions in $\mathrm{Ni}, \mathrm{Cr}$ and $\mathrm{Sc}_{\mathrm{c}}$ (table 4) suggest both olivine and clinopyroxene were the main fractionating phases in 
evolving the hawaiites. The alkali basalt patterns contrast markedly to the Tamerton Jurassic pattern, which shows lower LREE and MREE, but higher HREE, and deficiencies in $\mathrm{Nb}, \mathrm{Sr}, \mathrm{Ti}$; a minor Eu depletion suggests marked plagioclase crystallisation in its evolution (fig. 5), a feature not seen in Tasmanian Cenozoic basalts.

\section{Trace element ratios}

Most of the Tamar basalts show key trace element ratios that fall within the mantle array (Fitton et al. 1997) and within the range for HIMU (high $\mu$ ) - related Oceanic Island Basalt (OIB) suites (table 8, fig. 6). The HIMU-like signatures (Weaver 1991) occur among Tasmanian basalts and also in the Tasmanian seamounts off eastern Tasmania (Lanyon et al. 1993, Lanyon 1994, Crawford et al., 1997). These basalts represent a mantle source enriched in Ta and $\mathrm{Nb}$, relative to LREE, giving low $\mathrm{Zr} / \mathrm{Nb}$ ratios (av. $3.85 \pm$ 1.1) compared to other OIB sources. Part of this low $\mathrm{Zr} / \mathrm{Nb}$ signature, however, may relate to the degree of partial melting in the mantle sources, as $\mathrm{Zr} / \mathrm{Nb}$ increases with degree of saturation of the basaltic magmas (see table 8). Thus, it is important to compare similar ranges of basalt types. This may explain Tamar olivine nephelinite ratios that lie outside the Tasmanian seamount range where the lithology is largely based on basanites and alkali basalts. HIMU types also show relatively high values of $\mu$ (high ${ }^{238} \mathrm{U} / 204 \mathrm{~Pb}$ ratios) that produce high time-integrated $206 \mathrm{~Pb} / 208 \mathrm{~Pb}$ signatures $(>19)$, along with low ${ }^{87} \mathrm{Sr} /{ }^{86} \mathrm{Sr}$ and high ${ }^{143} \mathrm{Nd} /{ }^{144} \mathrm{Nd}$ values. No isotopic data are available for Tamar basalts, but the trace element ratios closely match basalts that carry such signatures at Weldborough and Table Cape (table 8). The origin of this HIMU imprint in southern Australasia and Antarctic basalts is uncertain, but may relate to mantle events around 80-100 Ma; it possibly stems from a large plume head or a series of small plumes developed with late Gondwanan break-up (see Lanyon et al. 1993, Lanyon 1994, Sutherland 1994, Crawford et al. 1997). The HIMU character in some basalts within Tasmania and in the Tasmanian seamounts (u 19-20.3; Ewart et al. 1988, Lanyon et al. 1993, Lanyon 1994, Crawford et al. 1997, Sutherland 2003, Zhang et al. 2006) becomes more pronounced in many Antarctic basalts ( $\mu$ > 20.5; Rochell et al. 1995, Panter et al. 2000). This has prompted suggestions that the HIMU-imprint may reflect variable subduction-related metasomatism of the underlying subcontinental lithospheric mantle (SCLM) during the Late Mesozoic (Finn et al. 2005).

HIMU signatures do not normally extend to tholeiitic lavas, and the Tamar tholeiites have distinctly different trace element ratios to the Tamar alkaline suites (fig. 8). The Kelso quartz tholeiites have $\mathrm{Zr} / \mathrm{Nb}$ ratios (10.9) that lie at the high end for Tasmanian Cenozoic tholeiites (Zhang et al. 2006), but with their $\mathrm{Ti} / \mathrm{V}$ ratios (63-67) remain quite distinct from the Tamarton tholeiite $(\mathrm{Zr} / \mathrm{Nb} 14.7, \mathrm{Ti} / \mathrm{V} 16)$ and typical Tasmanian Jurassic dolerites (Hergt et al. 1989).

\section{Mantle Sources}

The Tamar alkaline parental magmas mark several melting episodes derived from a garnet peridotite source based on its range of normalised trace element ratios $\left(\mathrm{La} / \mathrm{Nb}_{\mathrm{N}} 0.55-0.67\right.$; $\mathrm{Ce} / \mathrm{Y}_{\mathrm{N}}$ 5.52-12.0). This Tasmanian mantle source includes a HIMU imprint introduced before $47 \mathrm{Ma}$ (Rowella hawaiite).
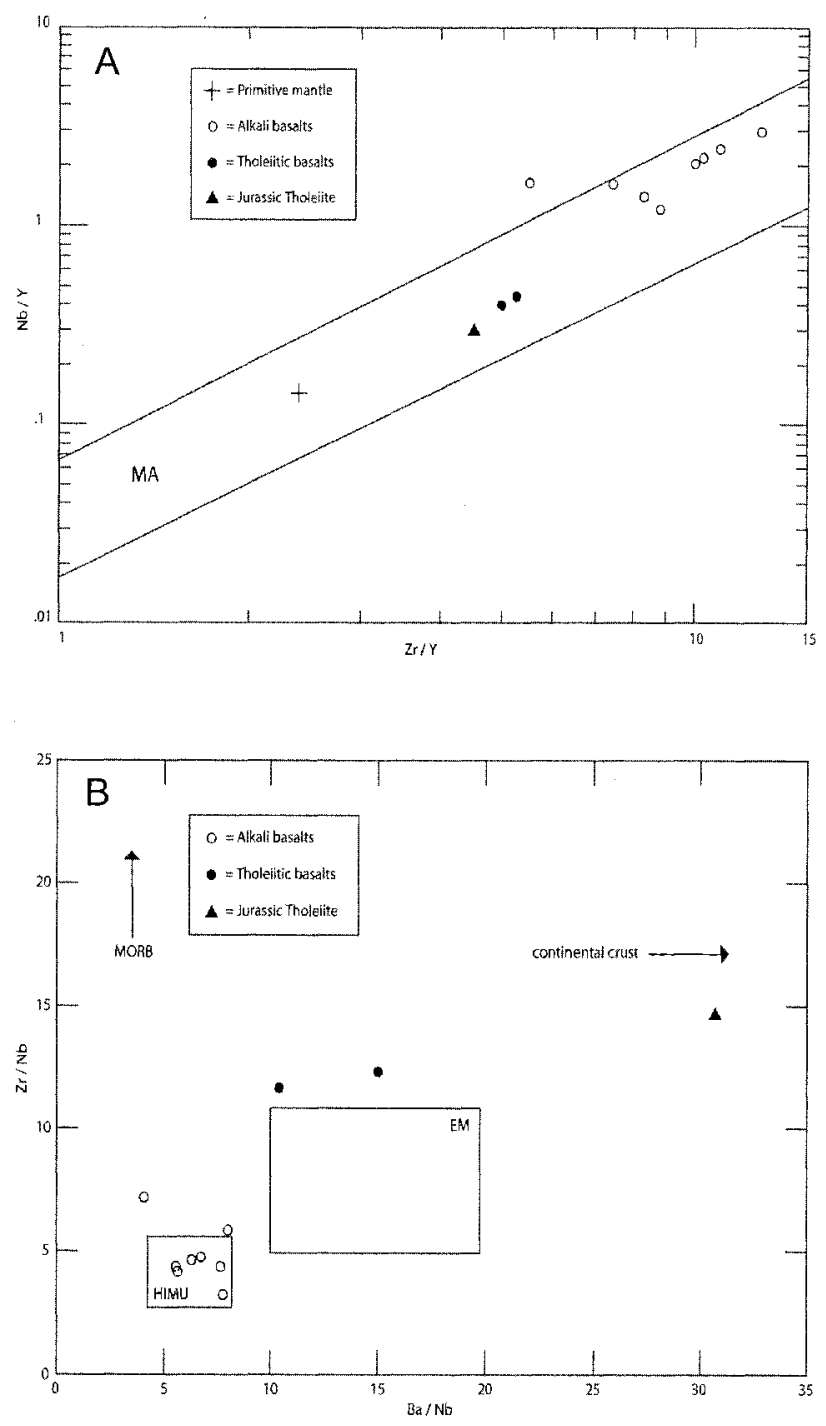

FIG. 6-Incompatible element ratio plots for Tamar basalts. (A) $N b / Y$ against $Z r / Y$ (MA Mantle Array; field after Fitton et al. 1997). (B) Zr/Nb against Ba/Nb (MORB Mid-Ocean Ridge Basalt, EM Enriched Mantle, HIMU High-mu mantle; fields after Weaver 1991).

More details of the peridotite source, its variations, and depths of magma generation for the HIMU-related Tamar basalts come from the more extended trace element data for the Rowella and Cocked Hat Hill basalts. The somewhat low $\mathrm{La} / \mathrm{Yb}_{\mathrm{N}}$ of these basalts $(9.5-10.4)$, within the primary alkaline range in Tasmania (8-42; Sutherland et al. 2004, Zhang et al. 2006), suggests between 10 to $20 \%$ degrees of partial mantle melting generated these voluminous Tamar magmas. Plots of $\mathrm{Ce} / \mathrm{Yb}_{\mathrm{N}}(7.7,9.6)$ against $\mathrm{Yb}_{\mathrm{N}}(9.24$; 8.71 ) for the two rocks suggest large clinopyroxene ( $\mathrm{cpx}$ ) to garnet (gnt) ratios were left in the residual source, between $14.0-14.5 \mathrm{cpx}$ and $1-0.5$ gnt based on such modelling by Jung \& Marsberg (1998).

Depths of magma segregations can be estimated using $\mathrm{Ce} /$ $\mathrm{Yb}$ and $\mathrm{Sm} / \mathrm{Yb}$ plots (Ellam 1992). In the Tamar hawaiites, $\mathrm{Ce} / \mathrm{Yb}(27.7,34.5)$ and $\mathrm{Sm} / \mathrm{Yb}(3.0,3.86)$ suggest magmas that rose from $70-90 \mathrm{~km}$ depth. The mantle regimes involved in basaltic melting can be discerned using $\mathrm{Nb} / \mathrm{La}$ to $\mathrm{La} / \mathrm{Yb}$ ratio plots (Abdel-Rahman 2002). The Tamar hawaiite 


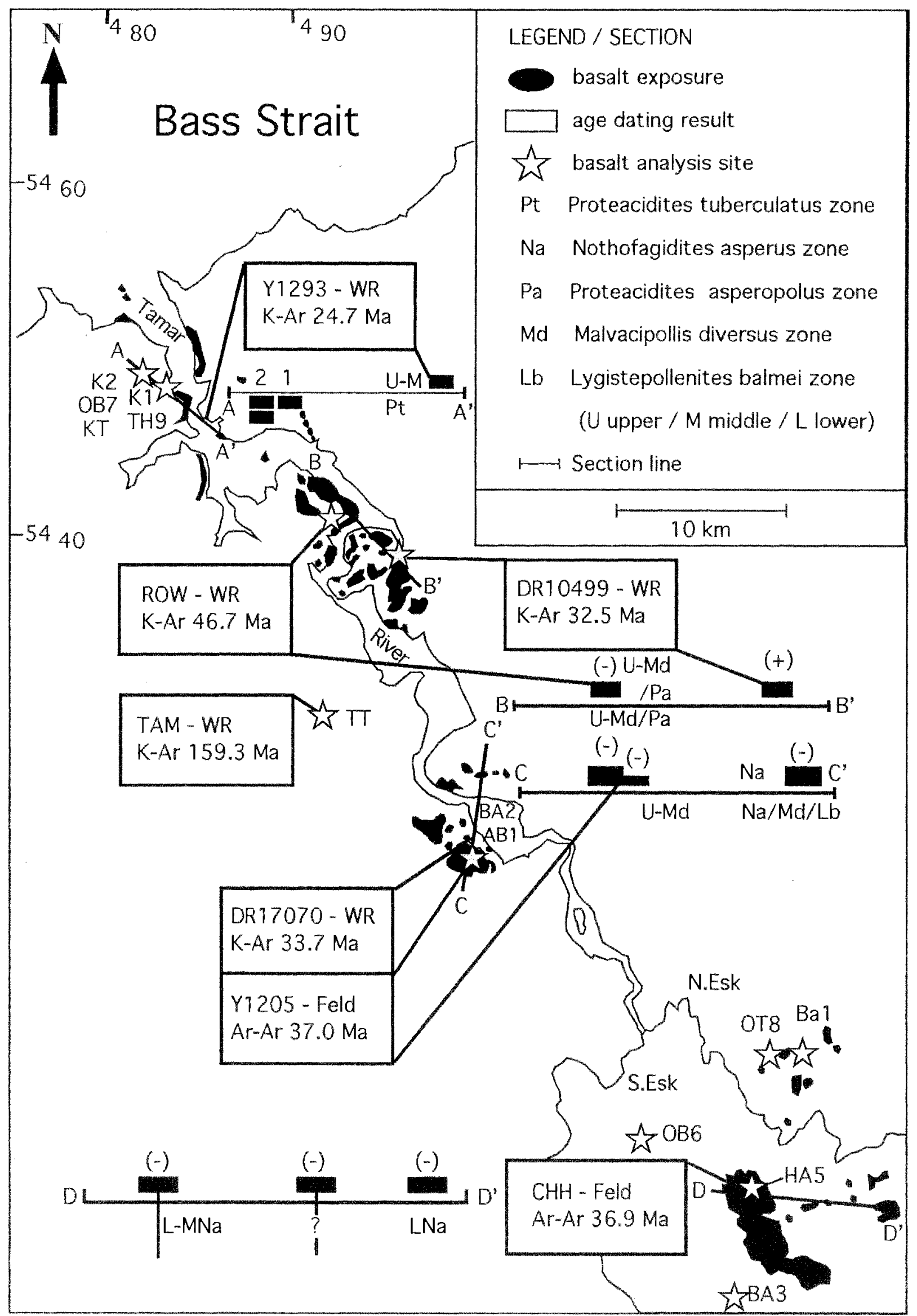

FIG. 7 - Dated and analysed basalt sites, Tamar areas, showing relationships to known palynology zones within sequences. Horizontal sections ( $\left.A-A^{\prime}, B-B^{\prime}, C-C^{\prime} D-D^{\prime}\right)$ are schematic cross-sections from the map. Analysed basalts are coded from the analytical tables and reversed (-) and normally (+) magnetised basalts are designated where known. 


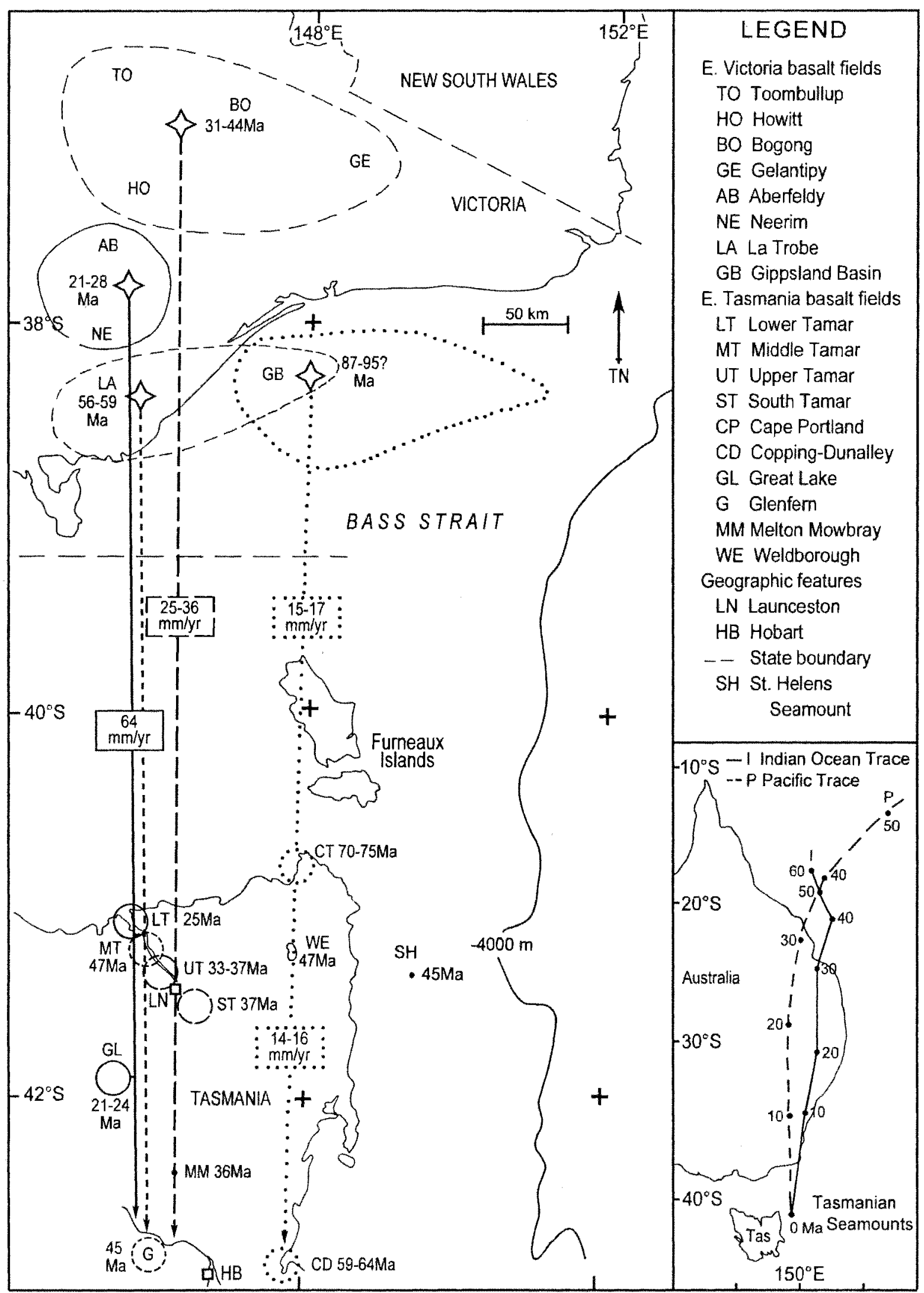

FIG. 8 - Tamar, east Tasmania and east Victoria basalt ages, related to migratory paths from Tasman margin plume sources (stars); ages come from Birch (2003), Sutherland et al. (2004), Everard et al. (2004a) and herein. Victorian magma sources and paths include Gippsland Basin (dotted lines), La Trobe (short dashed lines), North-east (long dashed lines), Centre-east (solid lines), with migration rates (boxes). Other Tasmanian basalt dates are left out for clarity. The depicted migration rates for each migration line are based on the Victorian-Tasmania basalt ages, for comparison with Australian plate motion paths for those times. The top inset lists basalt fields. The bottom inset shows Australian plate motion paths from $60 \mathrm{Ma}$ to a present Tasman Sea plume position, from Indian Ocean (I) and Pacific (P) hotspots. 
ratios $(\mathrm{Nb} / \mathrm{La} 1.65,1.75 ; \mathrm{La} / \mathrm{Yb} 14.0,15.5)$ suggest their magmas originated well within the asthenospheric field. Additional mantle affinities can be delineated by plotting $\mathrm{Ce} / \mathrm{Nb}$ against $\mathrm{Th} / \mathrm{Nb}$ ratios (Song et al. 2001). The Tamar hawaiite values (Ce/Nb 1.20, 1.28; Th/Nb 0.08, 0.07) place them with plume-related basalt magmas, close to the OIB field and Primitive Mantle ratios.

The Tamar olivine nephelinites are moderately depleted in $\mathrm{Rb}(20 \mathrm{ppm})$ and $\mathrm{Ba}(371 \mathrm{ppm})$ compared with some Tasmanian nephelinites (Rb 29-40, Ba 530-724 ppm). In this respect, they approach the Scottsdale nephelinite which is low in these elements relative to the standard HIMU component, possibly reflecting residual phlogopite in the source (see Sutherland et al. 2004). Whatever the precise origins of the HIMU- and garnet-bearing sources for the Tamar alkaline basalts, the Tamar tholeiites also reflect variations in source inputs. Tasmanian olivine tholeiites include co-existing types that differ in trace element and $\mathrm{Pb}$ isotope signatures, even at similar Mg-values and compatible element contents (Sutherland 2003, Sutherland et al. 2004). One group includes mantle-derived and near- HIMU Pb isotopic types and has higher $\mathrm{Rb}, \mathrm{Ba}$ and $\mathrm{Y}$ values similar to those shown by Tamar olivine tholeiites. Comparisons of the Tamar alkaline basalts with the adjacent Longford alkaline basalts (appendix B, fig. 4, table 8) show little differences in their basic geochemistry, although the Longford rocks tend to have relatively low $\mathrm{Na}_{2} \mathrm{O} / \mathrm{K}_{2} \mathrm{O}(2.0-2.3)$ and higher $\mathrm{Sr}$ (> $1000 \mathrm{ppm}$ ) values than their Tamar equivalents.

\section{Interpretive Model for Tamar Trough Evolution}

The new framework established for the Tamar Trough sequences, through combined palynological and basalt dating (fig. 7), allows further interpretations of its tectonic-thermal evolution. Initiated after Mid-Cretaceous to Early Palaeocene thermal uplift and rifting along the Bassian Tasman margin (Gleadow 1996, O'Sullivan et al. 2000, Cummins 2001), the Trough became a locus for sedimentation associated with the ancestral Tamar drainage. Volcanism did not begin until about $47 \mathrm{Ma}$ during the $P$. asperopolus Zone sedimentation, some 15-20 million years after rifting. Thus, the reasons behind the timing of volcanism need explaining beyond the Tamar faulting itself and consideration within the general Tasmanian-Bassian Cenozoic volcanism story.

During the early Tamar sedimentation, Tasmanian volcanism was largely restricted to the far eastern margin, between $70-76 \mathrm{Ma}$ in north-eastern Tasmania (Everard et al. 2004a, F.L. Sutherland and H. Zwingmann unpublished data) and between 59-65 $\mathrm{Ma}$ in south eastern Tasmania (Baillie 1987, Sutherland et al. 2004, Everard et al. 2004b). These dates imply potential southerly migratory volcanism, which back-tracks on slow Southern Ocean spreading rates to 85-95 Ma Gippsland Basin rifting and attendant Victorian margin volcanism (fig. 8). The Gippsland rift was a failed spreading arm of the Tasman Sea spreading triple point, a structure linked to plume volcanism (Sutherland 1994). The precise nature of this plume path and its geochemical relationships to the Tasmanian seamounts and Tasmanian basalts, however, have various interpretations (Lanyon et al. 1993, Crawford et al. 1997, Gaina et al. 2000, Sutherland et al. 2004). Nevertheless, the timing of HIMU-related alkali basalts (45-47 Ma) extending east from the Tamar Trough to Weldborough and St Helens Seamount and south to Glenfern in the Derwent Valley (Sutherland et al. 2004,
Everard et al. 2004b) is consistent with suggested Gippsland -Tasmanian Seamounts plume positions.

Recent studies of hotspot plumes, such as the Gippsland plume, suggest they may mark both metasomatic and magmatic processes related to triple junctions, failed rifts and ancient sutures, rather than necessitating deep-mantle plumes (Smith 1993, Finn et al. 2005, Sears et al. 2005). This is compatible with a proposed model for quasi-continuous, but episodic Tasmania volcanism that resulted from lithospheric drift of eastern Australia over an extended Tasman Sea plume zone (Sutherland et al. 1989). Furthermore, precise Tasmanian basalt ages (Sutherland et al. 2004, Everard et al. 2004b) and better-controlled Australian plate motion paths back to $80 \mathrm{Ma}$ (Sutherland 2003) now allow more detailed testing of the Tasman margin magma source model for Tasmanian volcanism. A fuller development will be given elsewhere, but for this Tamar Trough study a simplified model that relates the Tamar basalts to migratory links through eastern Victoria is presented in figure 8.

The proposed model predicts that the prominent Tamar alkaline volcanism dated at 46-47, 34-37 and 24-25 Ma and also the Early Oligocene tholeiites are a consequence of drift of the area over magma sources that previously fed the older eastern Victorian basalt fields. It also predicts the lack of Late Neogene ( $<24 \mathrm{Ma}$ ) volcanism in the Tamar sequence, as no basalt sources of appropriate age occur in eastern Victoria to intersect the Tamar track.

The periodic events where massive basalt flows entered the Tamar drainage between 47-24 (?) Ma, disrupted extant river courses and sedimentation regimes and also introduced resistant basalt bulwarks within the erosional cycles. In the last $20 \mathrm{Ma}$, the Tamar evolution has largely progressed through erosive/ sedimentary cycles linked to Tasmanian climatic and sea level changes, unimpeded by volcanic disruptions.

\section{ACKNOWLEDGEMENTS}

This study is dedicated to Walter Sutherland, former librarian, Launceston Regional Library, who greatly encouraged the earlier studies on the Tamar volcanic sequences by the first author. The present study initiated at the Australian Museum was approved by the then Director, Dr Michael Archer, with funding support from the Australian Museum Trust. Assistance with technical and script preparation came from Ross Pogson, Sue Folwell and Roger Springthorpe, Australian Museum. Samples for some of the analyses were provided by Noel Kemp, Geologist, Tasmanian Museum, Hobart. Basalt samples were prepared and analysed with the assistance of Les Hay, ChiefChemist, Jafar Taheri, Richie Woolley, Shane Heawood and David Shaw, Tasmanian Mineral Resources, with additional analyses by Maggie Loubser, Geology Department, University of Pretoria, and Andreas Spath, Geology Department, University of Cape Town, South Africa. Additional support for the project came from Peter Baillie, TGS-NOPEC Geophysical Company, West Perth, Western Australia. The script was read by Dr Larry Barron, Research Associate, Australian Museum. Constructive reviews were made by Drs Greg Jordan, School of Plant Science, University of Tasmania, Hobart, and Ian Roach, Department of Earth and Marine Sciences, Australian University, Canberra, ACT. S.M. Forsyth and J.L. Everard publish with permission of the Director of Mines, Mineral Resources Tasmania. 


\section{REFERENCES}

Abdel-Rahman, A.M. 2002: Mesozoic volcanism in the Middle East: geochemical, isotopic and petrogenetic evolution of extension-related alkali basalts from Central Lebanon. Geological Magazine 139: 621-640.

Adam, J. \& Green, T.H. 2006: Combined experimental and geochemical evidence for the origins of Tasmanian intraplate basales. 16 th Annual V.M. Goldschmidt Conference, Melbourne, Australia, 27 August-1 September, Abstracts.

Baillie, P.W. 1986: Radiometric age for the Circular Head and Green Hill basalts, north western Tasmania. Tasmania Department of Mines Report 1986/39 (unpubl.).

Baillie, P.W. 1987: A Palaeocene radiometric age for basalt at Bream Creek, south-eastern Tasmania. Tasmania Department of Mines Report 1987/21 (unpubl.).

Beccaluva, L., Siena, F., Coltorti, A., Digrande, A., Lo Giudice, A., Macciotta, G., Tassinari, R. \& Vaccaro, C. 1998: Nephelinitic to tholeiitic magma generation in a transtensional tectonic setting: an integrated model for the Iblean volcanism, Sicily. Journal of Petrology 39: 1547-1576.

Birch, W.D. (ed.) 2003: The Geology of Victoria. Geological Society of Australia Special Publication 23: 842 pp.

Bigwood, A.J., Forsyth, S.M. \& Hill, R.S. 1988: The Tamar Basin and Central Plateau. In Colhoun, E.A. (ed.): Cainozoic Vegetation of Tasmania. Newcastle University Special Publication: 132-143.

Blake, F. 1959: Geological Atlas 1 mile Series. Zone 7, Sheet No.47. Geological Survey of Tasmania Department of Mines

Blevin, J.E. (Compiler) 2003: Petroleum Geology of the Bass Basin-Interpretation Report, An Output of the Western Tasmanian Regional Minerals Program. Geoscience Australia, Record 2003/19.

Bonhomme, M.G., Thuizat, R., Pinault, Y., Clauer, N., Wendling, R.R. Winkler, R. 1975: Methode de datation potassium-argon. Appareillage et technique. University of Strasbourg, $53 \mathrm{pp}$.

Boynton, W.V. 1984: Geochemistry of the rare earth elements: meteorite studies. In Henderson, P. (ed.): Rare Earth Element Geochemistry. Elsevier, Amsterdam: 63-114.

Brown, A.V. \& Forsyth, S.M. 1984: Chemistry of Tertiary basalt and palynology of sediments from BHP drill holes, EL33/79. Tasmania Department of Mines Report 1984/39 (unpubl.).

Carey, S.W. 1946: Geology of the Launceston District. Records of the Queen Victoria Museum 2(1): 31-46.

Clark, D. \& Leonard, M. 2003: Principal stress orientations from multiple focal-plane solutions: new insights into the Australian intraplate stress field. Geological Society of Australia Special Publication 22 and Geological Society of America Special Paper 372: 91-105.

Clitheroe, G., Gudmundsson, O. \& Kennett, B.L.N. 2000: The crustal thickness of Australia. Journal of Geophysical Research 105: 13697-13713.

Crawford, A.J., Lanyon, R., Elmes, M. \& Eggins, S. 1997: Geochemistry and significance of basaltic rocks dredged from the South Tasman Rise and adjacent seamounts. Australian Journal of Earth Sciences 44: 621-632.

Cummings, A., Hillis, R. \& Tingate, P. 2001: Structural evolution and charge history in the Bass Basin-Preliminary results In Hill, K.C. \& Bernecker, T. (eds): Eastern Australasian Basins Symposium. Petroleum Exploration Society of Australia Special Publication: 645-648.

Cromer, W.C. 1980: A Late Eocene basalt from northern 'Tasmania. Search 11: 294-295.

Direen, N.G. \& Leaman, D.E. 1997: Geophysical modelling of structure and tectono-stratigraphic history of the Longford Basin, northern Tasmania. Exploration Geophysics 28: 29-33.
Ellam, R.M. 1992: Lithospheric thickness as a control on basalt geochemistry. Geology 20: 153-156.

Elliot, C.G., Woodward, N.B. \& Gray, D.R. 1993: Complex regional fault history of the Badger Head region, northern Tasmania. Australian Journal of Earth Sciences 40, 155-168.

Everard, J.L. 1990: Exploration Licence 99/87. Preliminary report for F. Bardenhagen. Two diamond-drill holes at Kelso, West Tamar. Tasmania Department of Resources and Energy, Division of Mines and Mineral Resources (unpubl. Report).

Everard, J.L. 2001: Inclusions of high pressure origin in Tasmanian Cenozoic basalts. A catalogue of localities. Tasmanian Geological Survey Record 2001/09.

Everard, J.L., Sutherland, F.L. \& Zwingmann, H. 2004a: A Cretaceous phonolite dyke from the Tomahawk River, Northeast Tasmania. Papers and Proceedings of the Royal Society of Tasmania 138: 11-33.

Everard, J., Zhang, M., Lo, C-H., O'Reilly, S. \& Forsyth, S. 2004b: Overview of Tasmania Tertiary basalts. Geological Society of Australia Abstracts Series 73:74

Ewart, A., Chappell, B.W. \& Menzies, M.A. 1988: An overview of the geochemical and isotopic characteristics of the eastern Australian Cainozoic volcanic provinces. In Menzies, M.A. \& Cox, E.G. (eds): Oceanic and Continental Lithosphere: Similarities and Differences. Journal of Petrology Special Lithosphere Issue: 225-274.

Finn, C.A., Muller, R.D. \& Panter, K.S. 2005: A Cenozoic diffuse alkaline magmatic province (DAMP) in the southwest Pacific without rift or plume origin. Geochemistry, Geophysics, Geosystems 6, Q 02005, doi: 10.029/2004 GC 000723.

Fitton, J.G., Saunders A.D., Norry, M.J., Hardason, B.S. \& Taylor, R.N. 1997: Thermal and chemical structure of the Iceland plume. Earth and Planetary Science Letters 153:197-208

Forsyth, S.M. 1989: The Tamar Graben. In Burrett, C.F. \& Martin, E.L. (eds): Geology and mineral resources of Tasmania. Geological Society of Australia Special Publication 15: 358-361.

Frey, F.A., Green, D.H. \& Roy, S.D. 1978: Integrated models of basalt petrogenesis: a study of quartz tholeiites to olivine melilitites from southeastern Australia utilizing geochemical and experimental petrological data. Journal of Petrology 19: 463-513.

Gaina, C., Muller, R.D. \& Cande, S. 2000: Absolute plate motion, mantle flow and volcanism at the boundary between the Pacific and Indian Ocean mande domains since $90 \mathrm{Ma}$. In Richards, M., Gordon, R.G. \& van der Hilst, R.D. (eds): The History and Dynamics of Global Plate Motions. American Geophysical Union Monograph 121: 189-210.

Gee, R.D. \& Legge, P.J. 1975: Geological Atlas 1 Mile series, Zone 7 Sheet No. 30 (8215N). Beaconsfield. Geological Survey of Tasmania Department of Mines.

Gleadow, A.J.W. 1996: Fission track thermochronology of southeastern Australia. Transactions of the Royal Society of Victoria 108 (2): 9-15.

Glenn, K.C., Willcox, B. \& Nicoll, R.S. 1999: Lord Howe Rise Regional Bizonation and Stratigraphy, 1999. Chart 26. Australian Geological Survey Organisation, Canberra.

Gulline, A.B. 1973: Geological Atlas 1 Mile series, Zone 7 Sheet No. 38 (8215S). Frankford. Geological Survey of Tasmania Department of Mines.

Hergt, J.M., Chappelle, B.W., McCulloch, M.T., McDougall, I. \& Chivas, A.R. 1989: Geochemical and isotopic constraints on the origin of the Jurassic dolerites of Tasmania. Journal of Petrology 30: 841-883.

Hess, J.C. \& Lippolt, H.J. 1994: Compilation of K-Ar measurements on HD-B1 standard biotite 1994 status report. Phanerozoic Time Scale. G.S. Odin, Paris. Bulletin of Liaison and Information, IUGS Subcommission of 
Geochronology 12: 19-23.

Heinrichs, H. \& Hermann, A.G. 1990: Praktikum der Analytischen Geochemie. Springler-Verlag, Berlin-Heidelberg: 669pp.

Hill, R.S. 1987: Discovery of Nothofagus fruits corresponding to an important Tertiary pollen type. Nature 327: 56-58.

Irvine, T.N. \& Baragar, W.R.A. 1971: A guide to the chemical classification of the common volcanic rocks. Canadian Journal of Earth Sciences 8: 523-548.

Johnson, R.W. 1989: Intraplate Volcanism in Eastern Australia and New Zealand. Cambridge University Press, Cambridge: $408 \mathrm{pp}$.

Jordan, G.L. \& Hill, R.S. 2002: Cenozoic plant macrofossil sites of Tasmania. Papers and Proceedings of the Royal Society of Tasmania 136: 127-139.

Jung, S. \& Masberg, P. 1998: Major- and trace-element systematics and isotope geochemistry of mafic volcanic rocks from Vogelsberg (central Germany). Constraints on the origin of continental alkaline and tholeiitic basalts and their mantle sources. Journal of Volcanology and Geothermal Research 86: 151-171

Kennett, B.L.N. 2003: Seismic structure in the mantle beneath Australia. Geological Society of Australia Special Publication 22 and Geological Society of America Special Paper 372: $7-23$.

Kennett B.L.N., Fishwick, S., Reading, A.M. \& Rawlinson, N. 2004: Contrasts in mantle structure beneath Australia: relation to Tasman Lines? Australian Journal of Earth Sciences 51: 563-569.

Lanyon, R. 1994: Mantle reservoirs and mafic magmatism associated with the break-up of Gondwana-The Balleny Plume and the Australian-Antarctic Discordance; U-Pb dating of a Proterozoic mafic dyke swarm in the Vestfold Hills, East Antarctica. Unpublished PhD thesis, University of Tasmania, Hobart.

Lanyon, R., Varne, R. \& Crawford, A.J. 1993: Tasmania Tertiary basalt, the Balleny Plume, and opening of the Tasman Sea (Southwest Pacific Ocean). Geology 21: 555-558.

Le Maitre, R.W. Ed. 1989: A Classification of Igneous Rocks and Glossary of Terms. Blackwell Scientific Publications, Oxford: 193pp.

Leaman, D.E., Baillie, P.W. \& Powell C.McA. 1994: PrecambrianTasmania: a thin -skinned devil. Exploration Geophysics 25, 19-23.

Leaman, D.E., Symonds, P.A. \& Shirley, J.E. 1973: Gravity survey of the Tamar region, northern Tasmania. Geological Survey of Tasmania Paper 1.

Longman, M.J., Matthews W.L. \& Rowe S.M. 1964: Geological Atlas One Mile series, K/55-7-39. Launceston. Geological Survey of Tasmania Department of Mines.

Ludwig, K.R. 2001: Isoplot/Ex.rev.2.49, a Geochronological toolkit for Microsoft Excel. Berkeley Geochronology Centre Special Publication 1a.

MacPhail, M.K. 1999: Palynostratigraphy of the Murray Basin, inland Southeastern Australia. Palynology 23: 197-240.

McClenaghan, M.P., Calver, C. R. \& Vicary, M.J. (comp.) 2005: Geology of Northeast Tasmania, Edition 2005.1. Geological Atlas 1: 250000 series. Mineral Resources Tasmania.

McClenaghan, M.P., Turner, N.J., Baillie, P.W., Brown, A.V., Williams, P.R. \& Moore, W.R. 1982: Geology of the Ringarooma-Boobyalla area. Bulletin Geological Survey of Tasmania 61.

McDougall, I. \& Harrison, T.M. 1999: Geochronology and Thermochronology by the ${ }^{40} \mathrm{Ar}{ }^{39} \mathrm{Ar}$ Method (2nd Edition). Oxford University Press, New York, 269 pp.

McDougall, I. \& Roksandic, Z. 1974: Total fusion ${ }^{40} \mathrm{Ar} /{ }^{39} \mathrm{Ar}$ ages using HIFAR reactor. Journal of the Geological Society of Australia 21: 81-89.

Matthews, W.L. 1974: Longford Basin Geology 1:100 000 map. Tasmania Department of Mines, Hobart.

Matthews, W.L. 1983: Geology and Groundwater Resources of the Longford Tertiary Basin. Bulletin Geological Survey of Tasmania 59.

Matthews, W.L. 1989: Longford sub-basin. In Burrett, C.F. \& Martin, E.L. (eds): Geology and mineral resources of Tasmania. Geological Society of Australia Special Publication 15: $370-372$.

Mazengarb, C. 2004: Map 5, Launceston-Potential Landslide Hazard-Tasmanian Landslide Hazard Series. Mineral Resources Tasmania, Dept. Infrastructure, Energy \& Resources, Hobart.

Montelli, R., Nolet, G., Dahlen, F.A., Masters, G., Engdahl, E.R. \& Hung, S-H. 2004: Finite-frequency tomography reveals on variety of plumes in the mantle. Science $\mathbf{3 0 3}$ : $338-343$.

Morrison, K.C. \& Davidson, J.K. 1989: In Burrett, C.F. \& Martin, E.L. (eds): Geology and mineral resources of Tasmania. Geological Society of Australia Special Publication 15: $347-356$

O'Neill, C.O., Moresi, L., Lenardic, A. \& Cooper, C.M. 2003: Inferences on Australia's heat flow and thermal structure from mantle convection modelling results. Geological Society of Australia Special Publication 22 and Geological Society of America Special Paper 372: 169-184.

O’Sullivan, P.B. \& Kohn, B.P. 1997: Apatite fission track thermochronology of Tasmania. Australian Geological Survey Record 1997/35.

O'Sullivan, P.B., Mitchell, M.M., O'Sullivan, A.J., Kohn, B.P. \& Gleadow, A.J.W. 2000: Thermotectonic history of the Bassian Rise, Australia: implications for the breakup of eastern Gondwana along Australia's southeastern margins. Earth and Planetary Science Letters 182: 31-47.

Panter, K.S., Hart, S.R., Kyle, P., Blusztanjin, J. \& Wilch, T. 2000: Geochemistry of Late Cenozoic basalts from the Crary Mountains: characterisation of mantle sources in Marie Byrd Land, Antarctica. Chemical Geology 165: 215-241.

Rawlinson, N., Houseman, G.A., Collins, C.D.N. \& Drummond B.J. 2001: New evidence of Tasmania's tectonic history from a novel seismic experiment. Geophysical Research Letters 28(17): 3337-3340.

Rawlinson, N. \& Kennett, B.L.N. 2006: Detailed teleseismic imaging of the crust and upper mantle beneath southeast Australia. $18^{\text {th }}$ Australian Earth Science Convention, 3-6 July, Melbourne, Extended Abstract, 5 pp. Australian Geological Sociery.

Reed, A.R., Calver, C. \& Bottrill, R. 2002; Palaeozoic suturing of eastern and western Tasmania in the West Tamar region: implications for the tectonic evolution of southeast Australia. Australian Journal of Earth Sciences 49: 809-830.

Rochell, A., Stein, M., Molzhan, M., Hart, S.R. \& Worner, G. 1995:Geochemical evolution of rift magmas by progressive tapping of a stratified mantle source beneath the Ross Sea Rift, Northern Victoria Land, Antarctica. Earth and Planetary Science Letters 131: 207-224.

Sears, J.W., Gregory, M.St. G. \& Winne, J.C. 2005: Continental rift systems and anorogenic magmatism. Lithos $\mathbf{8 0}$ : 147-154.

Seymour, D.B. 1989: Geological Atlas 1:50 000 Series. Sheet $36(8015 \mathrm{~N})$ St Valentines. Explanatory Report geological Survey Tasmania Department of Mines.

Smith, A.D. 1993: The continental mantle as a source for hotspot volcanism. Terra Nova 5: 452-460.

Song, X-Y., Zhou, M-F., Hou, Z-Q., Cao, Z-M., Wang, Y-L. \& Li, Y. 2001: Geochemical constraints on the mantle source of the Upper Permian Emeishan continental flood basalts, southwestern China. International Geology Review 43: 213-225.

Steiger, R.H. \& Jäger, E. 1977: Subcommission on Geochronology: convention on the use of decay constants in geo- and cosmochronology. Earth and Planetary Science Letters 
36: $359-362$.

Stover, E. \& Partridge, A.D. 1973: Tertiary spores and pollen from the Gippsland Basin, southeastern Australia. Proceedings of the Royal Society of Victoria 85: 237-286.

Sun, S-S. \& McDonough, W.F. 1989: Chemical and isotopic systematics of oceanic basalts; implications for composition and processes. In Saunders, A.D. \& Norry, M.J. (eds) Magmatism in the Ocean Basins. Geological Society of London Special Publication 42 (2):313-346.

Sutherland, F.L. 1969: The mineralogy, petrochemistry and magmatic history of the Tamar Lavas, northern Tasmania. Papers and Proceedings of the Royal Society of Tasmania 103: $17-34$ and 2 pages of plates.

Sutherland, F.L. 1971: The Geology and Petrology of the Tertiary Volcanic Rocks of the Tamar Trough, Northern Tasmania. Records of the Queen Victoria Museum 36: 58pp.

Sutherland, F.L. 1994: Tasman Sea evolution and hotspot trails. In Van der Lingen, G.J., Swanson, K.M. \& Muir, R.J. (eds): Evolution of the Tasman Sea Margin. A.A. Balkema Rotterdam: 35-51.

Sutherland, F.L. 2003: 'Boomerang' migratory intraplate Cenozoic volcanism, eastern Australian rift margins and the IndianPacific mantle boundary. Geological Society of Australia Special Publication 22 and Geological Society of America Special Paper 372: 203-221.

Sutherland, F.L. \& Wellman, P. 1986: Potassium-argon ages of Tertiary volcanic rocks, Tasmania. Papers and Proceedings of the Royal Society of Tasmania 120: 77-86.

Sutherland, F.L., Forsyth, S.M. \& Zwingmann, H. 2002: Bassian basalts: Daring, Cenozoic biogeohistory and a new model for Tasmanian volcanism. Geological Society of Australia Abstracts Series 67: 251.

Sutherland, F.L., Ewart, A., Raynor, L.R., Hollis, J.D. \& McDonough, W.D. 1989: Tertiary basaltic magmas and the Tasmanian lithosphere. In Burrett, C.F. \& Martin, E.I. (eds): Geology and Mineral Resources of Tasmania.
Geological Society of Australia Special Publication 15 386-389.

Sutherland, F.L., Graham, I.T., Everard, J.L., Forsyth, S.M. \& Zwingmann, H. 2004: Cenozoic basalts, Tasmania: Landscapes, exposures, ages, petrography, geochemistry, entrainments and petrogenesis. 17 th Australian Geological Convention, February 2004, Tasmania, Field Guide A5. Geological Society of Australia, Sydney: 58 pp.

Sutherland, F.L., Hendry, D.F., Barron, B.J., Matthews, W.L. \& Hollis, J.D. 1996: An Unusual Tasmanian Tertiary Basalt Sequence, Near Boat Harbour, Northwest Tasmania. Records of the Australian Museum, 48: 131-161.

Turner, N.J. 1975: Stratigraphy and landslips in the Craigburn and Beauty Point areas. Tasmanian Department of Mines Report 1975/79 (unpublished).

Weaver, B.L. 1991: Trace element evidence for the origin of ocean-island basalts. Geology 19: 123-126.

Wells, P.M. 1988: Palynology of Tertiary sediments from Windemere Drill Holes 1 and 3. Tasmanian Depariment of Mines Report 1988/05 (unpublished).

Williams, E. 1979: Tasman Fold Belt System in Tasmania: Explanatory Notes for the 1: 500000 Structural Map of Pre- Carboniferous Rocks of Tasmania (revised edition). Tasmanian Department of Mines, Hobart.

Zhang, M., O'Reilly, S.Y. \& Everard, J.L. 2006: Trace clement geochemistry, Radiogenic isotopic characteristics, Mantle sources and petrogenesis. In Corbetr, K. \& Quilty, P. (eds). The Geological Evolution of Tasmania, in press.

Zhou, H., Zindel, A., Xu, X. \& Qi, Q. 2000: Major, trace element, and $\mathrm{Nd}, \mathrm{Sr}$ and $\mathrm{Pb}$ isotope studies of Cenozoic basalts in SE China: mantle sources, regional variations, and tectonic significance. Chemical Geology 171: 33-47.

(accepted 2 October 2006

\section{APPENDIX A \\ Petrographic description of analysed, dated and new Tamar Trough basalts}

\author{
Quartz tholeiite: West of Tamerton, Loira (DQ 920302) (DR \\ 17074). \\ See plate $2 \mathrm{~F}$
}

\section{Quartz tholeiite: Kelso DDH 1/51.55m (DQ 819489).}

Olivine phenocrysts $(5-10 \% \leq 1 \mathrm{~mm}$ but mostly $-500 \mu \mathrm{m})$ grade into an intergranular to intersertal groundmass, with patches of "black glass". Most glass areas are completely altered to pale brown, coarsely fibrous material, although some remain largely fresh.

Groundmass plagioclase laths range from microphenocrysts ( $\leq$ $700 \times 150 \mu \mathrm{m})$ to acicular laths $(-100 \mu \mathrm{m}$ long, $200-300 \mu \mathrm{m}$ long) and are accompanied by interstitial granules of colourless augite (50-100 $\mu \mathrm{m})$, subordinate olivine, and a black glassy mesostasis, partly resolvable into a dense dissemination of blebs and microlites of an opaque mineral.

Quartz tholeiite: Kelso DDH1 (DQ 819489) (G400356).

Fresh, equant olivine crystals (up to $1 \mathrm{~mm}$ ), and less abundant plagioclase in narrow laths (typically 300-500 x 50-100 $\mu \mathrm{m}$, but grading down to microlites) are set in an abundant mostly clear glass $(70-80 \%)$. The pale to yellow-green unaltered glass in places passes into a turbid, dark orange-brown to nearly opaque material. Between fresh and unaltered glass, plagioclase laths are surrounded by brown altered glass and probably acted as nuclei during the alteration of glass. A few irregular vesicles and amygdales contain very fine-grained, brownish material.

\section{Quartz tholeiite: Kelso 2/27.5 m (DQ 814491).}

Numerous polygonal euhedra and subhedra of olivine (mostly 500 $\mu \mathrm{m}-1 \mathrm{~mm}$ across) and plagioclase laths $(\leq 1 \mathrm{~mm} \times 150 \mu \mathrm{m})$ grade down into a groundmass in which inhomogeneous black glass contains elongate to radiating trichites of plagioclase and uneven disseminations of opaque dust. Numerous irregular vesicles ( $\leq 10$ $\mathrm{mm}$ ) are lined with fibrous zeolite (phillipsite?) or pale yellowbrown, isotropic to kelyphytic material (palagonite?); some contain carbonate.

\section{Transitional olivine basalt: Kelso DDH 2/7.0 m (DQ} 814491).

Numerous, mostly corroded and partly altered, olivine phenocrysts ( $\leq$ $1 \mathrm{~mm}$ ) lie in a medium- to fine-grained holocrystalline intergranular groundmass with a marked flow lamination.

The groundmass contains aligned plagioclase laths $(200-400$ $\mu \mathrm{m}$ long), interstitial granules of colourless augite $(10-50 \mu \mathrm{m})$, abundant well-crystallized opaque minerals in equant grains (10-40 $\mu \mathrm{m})$ and acicular laths $(\leq 100 \times 5 \mu \mathrm{m})$ and a mesostasis enclosing tiny needles of apatite. Sparse, nearly round amyglades $(\leq 1 \mathrm{~mm})$ contain granular carbonate. 
Olivine basalt: Prospect Vale, New Bass Highway (EQ 106068) (L 10).

See plate $2 \mathrm{~A}$

Hawaiite: Cocked Hat Hill (EQ 16810365) (R010199).

Relatively abundant olivine phenocrysts ( $\leq 3.5 \mathrm{~mm}$, but mostly $500 \mu \mathrm{m}-1 \mathrm{~mm}$ ) and rare glomerocrysts grade down into a fairly coarse-grained intergranular groundmass consisting of plagioclase in laths and irregular anhedra (mostly $500 \mu \mathrm{m}-1 \mathrm{~mm}$ long), titaniferous augite granules (100-200 $\mu$ macross), elongate (100-400 $\mathrm{x} 10-20 \mu \mathrm{m})$ opaque grains and tiny biotite flakes $(\leq 50 \mu \mathrm{m})$ and acicular apatite. A few rounded amygdales $(\leq 300 \mu \mathrm{m})$ are filled with nepheline. Some alteration appears on margins and fractures of olivine phenocrysts along with similar parchy material in the groundmass.

\section{Hawaiite: South Esk River, near Sawpit Ford (EP 16439505)} (R0011953, PTH 3).

Subhedral olivine phenocrysts (mostly $400 \mu \mathrm{m}-1 \mathrm{~mm}$, rarely $\leq 1.5$ $\mathrm{mm}$ across) and glomerocrysts are partly altered to "iddingsite." The coarse-grained intergranular groundmass consists of plagioclase laths $(500 \mu \mathrm{m}-1 \mathrm{~mm})$ and tabular zoned sections, titaniferous augite granules $(\leq 150 \mu \mathrm{m})$ and elongate $(150-400 \times 10-20 \mu \mathrm{m})$ to equant angular opaque grains. Sparse patches of indeterminate mesostasis contain yellow-hrown alteration products and opaque dust.

\section{Hawaiite: South Esk River, near "Leighlands" (EP 17006451)} (R01 1954, PTH4).

Olivine phenocrysts $(500 \mu \mathrm{m}-1 \mathrm{~mm})$ lie in an intergranular to intersertal groundmass of plagioclase laths $(1 \mathrm{~mm})$, titaniferous augite granules and a turbid mesostasis which includes acicular opaque grains ( $\leq 200 \mu \mathrm{m}$ long), titaniferous augite, low birefringence phases and brownish alterations and approaches "black glass."
Hawaiite: South Esk River, near "Rosebank" (EP 17109341) (R01 1955, PTH 5).

Partly altered olivine crystals and phenocrysts $(500 \mu \mathrm{m}-1 \mathrm{~mm})$ lie in an intergranular groundmass of plagioclase laths $(500 \mu \mathrm{m}-1 \mathrm{~mm})$, titaniferous augite granules and elongate opaque grains. Small (500 $\mu \mathrm{m}-1 \mathrm{~mm}$ ) segregations and anygdales are filled with feldspathoidal or zeolitic material.

Hawaiite: Rowella DDH 2, 117.08 m (DQ 930413) (CHH). See plate $2 \mathrm{E}$

\section{Alkali basalt: Rosevears Estate Vineyard, Strathlyn (EQ 007233) (DR 17385).}

See plate $2 B$

Basanite: Rosevears Estate Vineyard, Strathlyn (EQ 006233) (DR 17384).

See plate $2 \mathrm{C}$

\section{Basanite: Midland Highway bridge, Perth (EP 152962) (R010199, CHH).}

Microphenocrysts of olivine ( $20 \%$ ) up to $800 \mathrm{um}$ across, but mostly $\leq 500 \mu \mathrm{m}$, accompany subordinate microphenocrysts of ritaniferous augite (up to $2 \mathrm{~mm}$ across, but commonly $\leq 1 \mathrm{~mm}$ ), which in places form glomerocrysts or rosettes.

The intergranular groundmass contains granules of titaniferous augite (typically $50-200 \mu \mathrm{m}$ across), some sparse plagioclase laths (typically $150-600 \times 15-50 \mu \mathrm{m}$ ) and equant angular opaque grains (typically $40-120 \mu$ macross). A low birefringence mesostasis contains zoned alkali feldspar and clear nepheline. A few small segregations contain mosaics of nepheline and very finely acicular apatite. An amygdale $1.5 \mathrm{~mm}$ across contains radiating sheaves of a zeolite (?) mineral, possibly phillipsite.

APPENDIX B

Whole rock XRF element analyses, Longford Basin basalts

\begin{tabular}{|c|c|c|c|c|c|c|c|}
\hline \multicolumn{4}{|c|}{ Major oxides } & \multicolumn{4}{|c|}{ Trace elements } \\
\hline wt $\%$ & $\mathrm{~L}, 1$ & L2 & L3 & $\mathrm{ppm}$ & L1 & $\overline{\mathrm{L} 2}$ & L3 \\
\hline $\mathrm{SiO}_{2}$ & 46.14 & 45.82 & 47.07 & $\mathrm{Ni}$ & 240 & 210 & 240 \\
\hline $\mathrm{TiO}_{2}$ & 2.18 & 2.18 & 1.98 & $\mathrm{Cr}$ & 270 & 280 & 290 \\
\hline $\mathrm{Al}_{2} \mathrm{O}_{3}$ & 14.20 & 13.99 & 14.20 & $\mathrm{Co}$ & 58 & 55 & 55 \\
\hline $\mathrm{Fe}_{2} \mathrm{O}_{3}^{3}$ & 3.01 & 2.30 & 3.10 & Sc & 20 & 21 & 20 \\
\hline $\mathrm{FeO}$ & 8.60 & 9.37 & 8.15 & V & 130 & 125 & 120 \\
\hline $\mathrm{MnO}$ & 0.17 & 0.17 & 0.17 & $\mathrm{Ba}$ & 370 & 310 & 280 \\
\hline $\mathrm{MgO}$ & 9.65 & 10.22 & 9.74 & $\mathrm{Rb}$ & 23 & 23 & 22 \\
\hline $\mathrm{CaO}$ & 9.37 & 9.36 & 8.79 & $\mathrm{Sr}$ & 1300 & 780 & 1000 \\
\hline $\mathrm{Na}_{2} \mathrm{O}$ & 2.54 & 2.65 & 2.75 & Y & 23 & 21 & 24 \\
\hline $\mathrm{K}_{2} \mathrm{O}$ & 1.29 & 1.20 & 1.19 & $\mathrm{Zr}$ & 230 & 210 & 200 \\
\hline $\mathrm{P}_{2}^{2} \mathrm{O}_{5}$ & 0.55 & 0.56 & 0.45 & $\mathrm{Nb}$ & 47 & 51 & 41 \\
\hline $\mathrm{H}_{2}^{2} \mathrm{O}_{+}$ & 1.85 & 1.76 & 1.92 & $\mathrm{La}$ & 29 & 27 & 27 \\
\hline $\mathrm{CO}_{2}$ & 0.06 & 0.05 & 0.15 & $\mathrm{Ce}$ & 75 & 74 & 59 \\
\hline \multirow[t]{2}{*}{$\mathrm{SO}_{3}^{2}$} & 0.00 & 0.01 & 0.01 & $\mathrm{Nd}$ & 30 & 38 & 34 \\
\hline & & & & $\mathrm{Pb}$ & $<10$ & $<] 0$ & $<10$ \\
\hline \multirow[t]{2}{*}{ Total } & 99.61 & 99.64 & 99.67 & Th & $<10$ & $<10$ & $<10$ \\
\hline & & & & $\mathrm{U}$ & $<10$ & $<10$ & $<10$ \\
\hline $\mathrm{Na}_{2} \mathrm{O} / \mathrm{K}_{2} \mathrm{O}$ & 1.97 & 2.21 & 2.31 & $\mathrm{Cu}$ & 59 & 59 & 54 \\
\hline Mg value* & 64.21 & 65.27 & 65.20 & $\mathrm{Zn}_{\mathrm{n}}$ & 120 & 115 & 120 \\
\hline $\mathrm{An} \% *$ & 51.00 & 51.59 & 48.16 & $\mathrm{Ga}$ & 21 & 22 & 21 \\
\hline D.I.* & 29.72 & 29.09 & 31.04 & ¿ Inc. & 588 & 566 & 605 \\
\hline
\end{tabular}

* Calculated at $\mathrm{Fe}_{2} \mathrm{O}_{3} / \mathrm{FeO} \sim 0.2$. D.I. $=\Sigma$ or, ab, ne. $\Sigma$ Inc. $=\Sigma \mathrm{Ni}, \mathrm{Cr}, \mathrm{Co}, \mathrm{Sc}$.

L1. Alkali olivine basalt. "Karalta", Pateena Road, Longford (EP 09878818).

L.2. Alkali olivine basalt. "Glendessary" via Evandale, Longford (EP 18849729).

L3. Transitional olivine basalt. Rail cutting, E of Longford, Longford (EP10929628). 


\section{ROYAL SOCIETY OF TASMANIA PAPERS AND PROCEEDINGS GUIDE TO AUTHORS}

The Royal Society of Tasmania, which was founded in 1843 , is the oldest scientific society in Australia and New Zealand and the third oldest Royal Society in the Commonwealth. Since 1849, it has published annual volumes of refereed scholarly papers about Tasmania in the Papers and Proceedings of the Royal Society of Tasmania and, from time to time, monographs and special, generally thematic, issues of the journal. Its subscribers include learned societies, research institutions and universities all over the world.

\section{Scope}

Papers and Proceedings of the Royal Society of Tasmania publishes research that focuses on Tasmania or is particularly relevant to Tasmanians. Although the subject matter is usually within the natural sciences or history, papers in the arts, social sciences or other disciplines are encouraged. Papers should embody the results of a significant piece of original work or offer an original synthesis or review.

Papers may be submitted by members of the Society or by non-members. Submission implies that the contents are original and have not been published previously in any form, and that no similar manuscript will be submitted elsewhere.

\section{Copyright}

Verbatim quotations of text must be acknowledged and the source given (with the relevant page number/s). Authors must specifically acknowledge the source of illustrations that have been previously published elsewhere and are not their property, and must obtain permission for publication of text or illustrations covered by copyright. Permission to use material previously published by the Royal Society of Tasmania can be obtained upon application to the Council.

\section{Address for Submissions}

Honorary Editor, The Royal Society of Tasmania, GPO Box 1166, Hobart, Tas 7001, Australia.

Please include your email address and the name of the person the editor can contact with editorial queries, proofs etc. Also note whether electronic versions of the text of the paper (preferably in Word) and of the figures (in TIF, JPG PDF or EPS) are available. Please submit an electronic version with your hard copy of expedite transmission to referees.

\section{Refereeing Policy}

The Council of the Society will take into consideration the advice of at least two reviewers before deciding for or against accepting the paper for publication. That decision will be final. If the paper is not accepted, one copy will be retained by the Society, the others returned to the author. Once accepted, papers and their copyright belong to the Society. However, an author may copy his or her paper for distribution as a reprint, and reproduction of synopses in abstracting journals is authorised. PDF files are available for purchase by authors.

\section{Style}

Referees are not expected to correct grammatical or spelling mistakes; papers should be well written, with the minimum of jargon. Remember that many of the readers of this journal are not specialists in your field; do not exclude them.

The Editor reserves the right to adjust the text for clarity, conformity to style, and publication standards. In general, the editor follows the conventions in the current edition of the Style Manual for Authors, Editors and Printers published by the Australian Government Publishing Service, and the spelling in the Macquarie Dictionary.

\section{Text format}

- use 11 or 12 point serif type

- print double-spaced (not one-and-a-half spaced)

- do not justify (i.e. use ragged right)

- number all pages consecutively, including references, figure legends, appendices

- number every fifth line (automatic in Word) to assist reviewers

- send three copies of the paper (including good copies of the illustrations)

- note whether electronic versions of the text (Word) and figures (TIF, JPG, PDF or EPS) are available

- supply key words $(2-10)$ for the use in abstracting journals

- include an abstract of no more than 200 words

Citation format

- published material e.g., Brown (1983), Brown \& Green (1985), Brown etal. (1987), Brown etal. (in press), (Brown 1983)

- note any information supplied personally to the author(s) e.g., (T.O. White pers. comm.); do not include in the list of references; obtain permission from the informant (preferably in writing) to quote him or her

- include the relevant page number of a lengthy cited work e.g., (1983, p. 110)

- do not refer to papers "in preparation" without an explanatory note to the editor

\section{References format}

The reference list should conform to the format used in recent volumes of the journal, with the exception of three minor changes:

- journal titles to be given in full (not abbreviated)

- book titles to be in upper and lower case, italicised (not all-capitals)

- Authors names should be in upper and lower case bold face

Tables and Illustrations

- number consecutively in the order of the first reference to them in the text 


\section{Tables}

The preferred dimensions for tables are either (a) 65 lines or less in depth, each line of no more than 130 characters and spaces in length, or (b) 42 lines or less in depth, each line of no more than 190 characters and spaces. These dimensions include the title, explanatory material and column headings.

- tables should be "stand-alone", with sufficient information for the table to be understood without reference to the text

- explain symbols and abbreviations (e.g., n.d. = no data) in the footnotes to the table

- should have the fewest possible lines; do not use the grid "default" in Word

\section{Illustrations (figures and plates)}

The maximum dimensions for illustrations, when printed, are 250 by $167 \mathrm{~mm}$, including captions. Wherever possible, illustrations will be fitted into a single column (width 80 $\mathrm{mm})$. Illustrations:

- preferably in black and white; colour illustrations may be used but authors will be expected to bear the extra cost of printing

- supply captions for all illustrations

- use keys within the artwork to explain symbols used in the illustrations

- add bar scales or objects of known or stated dimensions to show the scale of illustrations wherever appropriate

- write the author's name and the figure or plate number in soft pencil on the back of each illustration

- lines, letters and symbols must be of a size and weight to stand the likely reduction to the printed size without breaking up, becoming too faint, filling in or becoming too small for ready legibility; the smallest letter or number; after reduction, should be not less than $2 \mathrm{~mm}$ high and, on the axes of graphs, 2.5 or $3 \mathrm{~mm}$ high.

- electronic figures should be made available in TIF, JPG, EPS or PDF formats. DO NOT EMBED ILLUSTRATIONS (FIGURES AND PLATES) IN A WORD DOCUMENT.

\section{Appendices}

Material presented as Appendices (labelled A, B, C, etc.) are accepted at the editor's discretion, but may be printed in a smaller type than the body of the paper.

\section{Conventions}

The Botanical, Zoological and Stratigraphic Codes of Nomenclature should be fully observed.

Use of SI units of measurement is strongly preferred where consistent with the means of measurement.

\section{Costs}

To keep publication costs down, texts should be concise, and tables and illustration should be used sparingly, both for economy and for maximum effect in the published form. Grants from author's institutions or elsewhere to support publication should be secured if possible. Authors are responsible for the costs of changes, other than minor corrections, to proofs. Page charges will normally be levied on the eleventh and each subsequent page of a paper as printed. The author will be charged the cost of printing colour illustrations. 